\title{
Cien años haciendo ciencia: la Cátedra de Hidrología Médica de la Facultad de Medicina de la Universidad Complutense (1912-2012). Catálogo de la exposición
}

\author{
Juan Antonio RODRÍGUEZ SÁNCHEZ ${ }^{(1)}$ \\ Javier DE JORGE GARCÍA-REYES ${ }^{(2)}$ \\ Manuela CREGO CASTAÑO ${ }^{(2)}$ \\ ${ }^{(1)}$ Área de Historia de la Ciencia. Facultad de Medicina. Universidad de Salamanca \\ ${ }^{(1)}$ Biblioteca. Facultad de Medicina. Universidad Complutense Madrid \\ jarshm@usal.es, jjorge@buc.ucm.es, mcrego@buc.ucm.es
}

\begin{abstract}
Resumen
Con motivo de la celebración del primer centenario de la creación de la Cátedra de Hidrología Médica en la Universidad Central (hoy Universidad Complutense de Madrid) se organizó en la Facultad de Medicina, de abril a junio de 2012, una exposición que reúne libros, memorias manuscritas, documentos administrativos, planos, fotografías y objetos que permiten exhibir las circunstancias que concurrieron para la creación de dicha cátedra, su evolución y principales aportaciones, así como su presente y retos futuros. La exposición física se ha acompañado de una versión virtual expandida que garantiza su accesibilidad permanente y la incorporación de nuevos documentos.
\end{abstract}

Palabras clave: Hidrología Médica, Exposiciones, Bibliografía

\author{
One hundred years making science: the Chair of Medical Hydrology \\ in the Faculty of Medicine, Complutense University (1912-2012). \\ Exhibition catalog
}

\begin{abstract}
On the occasion of the first century's anniversary of the creation of the Chair of Medical Hydrology in the Central University (now so-called University Complutense of Madrid) was organized at the Faculty of Medicine, from April to June 2012, an exhibition of books, handwritten memories, administrative documents, drawings, photographs and objects that allow displaying the circumstances surrounding the creation of this chair, its evolution and main contributions, as well as its present and future challenges. Physical exposure is accom-
\end{abstract}


panied by an expanded virtual version that guarantees permanent access and the addition of new documents.

Key words: Medical Hidrology, Exhibitions, Bibliography

\section{REFERENCIA NORMALIZADA}

Rodríguez-Sánchez JA, De Jorge J, Crego M. Cien años haciendo ciencia: la Cátedra de Hidrología Médica de la Faculta de Medicina de la Universidad Complutense (1912-2012). Catálogo de la exposición. Balnea, 2012, 7, 263-308.

Exposición virtual disponible en: http://www.ucm.es/BUCM/med/51068.php

\section{SUMARIO}

Introducción. 1. Los antecedents: primeros tratadistas médicos. 2. La obra del Cuerpo de Médicos de Baños (1816-1877). 3. La Sociedad Española de Hidrología Médica: el proceso de institucionalización de la disciplina. 4. La legitimación por el pasado: bibliografía y memoria. 5. Sistematizar conocimientos: en busca de un tratado. 6. La industria balnearia. 7. La guía del bañista. 8. La creación de la cátedra de Hidrología Médica. 8.1. La enseñanza de la Hidrología Médica antes de la cátedra. 8.2. Hipólito Rodríguez Pinilla: la creación del corpus teórico hidrológico. 8.3. Hipólito Rodríguez Pinilla, médico de baños. 8.4. Los primeros discípulos: José García Viñals. 9. Guerra, depuración y primer franquismo. 9.1. El contexto balneario: cambios sociales, cambios administrativos. 9.2. Vicisitudes de la Cátedra. 9.3. Entre la SEHM y la Cátedra. 10. José San Román y el "Instituto Alfonso Limón Montero". 11. Transiciones: Antonio Castillo de Lucas. 12. Nuevas orientaciones: Manuel Armijo Valenzuela y la Escuela de Hidrología Médica. 13. Josefina San Martín Bacaicoa: internacionalización. 14. La docencia de la Hidrología Médica: de la estructura departamental a los retos del Espacio Europeo. 14.1. Francisco Maraver Eyzaguirre: relaciones institucionalizadas. 14.2. Francisco Armijo Castro: nuevas técnicas analíticas. 15. La contribución a la Hidrología desde otras ciencias. 15.1. El misterio de las aguas mineromedicinales: la Química. 15.2. La comprensión del terreno: la Geología y las aguas minero-medicinales. 15.3. El agua minero-medicinal como fármaco. 16. La exposición virtual

\section{INTRODUCCIÓN}

Con motivo de conmemorarse el centenario de la creación de la Cátedra de Hidrología Médica en la entonces Universidad Central, se ha inaugurado, en la Facultad de Medicina, una exposición de documentos que pretenden ayudar a la comprensión de cómo se creó, cuál ha sido su evolución y qué importancia tiene. Bibliografía, manuscritos, fotografías y diversos objetos ilustran ese pasado hasta el presente.

Auspiciada por una comisión coordinada por la profesora emérita Josefina San Martín Bacaicoa, catedrática de la disciplina, la exposición ha estado dirigida en sus aspectos conceptuales por Juan Antonio Rodríguez Sánchez, profesor de Historia de la Ciencia de la Universidad de Salamanca, y en los técnicos por Javier de Jorge García-Reyes, director de la Biblioteca de la Facultad de Medicina de la Universidad Complutense. La versión virtual de la misma, que permite hacer de ella una 
exposición permanente en red, ha estado a cargo de Manuela Crego, subdirectora de la Biblioteca de la Facultad de Medicina de la Universidad Complutense.

Se exhiben libros del rico patrimonio de la Universidad Complutense, principalmente los de la Biblioteca de la Facultad de Medicina, que posee una colección única de bibliografía hidrológica y memorias de médicos de baños, impresas y manuscritas. Las limitaciones impuestas por la seguridad y conservación de algunos textos (incunables y obras del siglo XVI y XVII) han impedido su exhibición, aunque están presentes en la versión virtual. Igualmente, han sido estos criterios los que han impedido incorporar documentos procedentes de archivos estatales, si bien se ha contado con los de la propia Facultad de Medicina.

Han resultado de inestimable ayuda las aportaciones realizadas por la profesora Josefina San Martín y los profesores Francisco Maraver Eyzaguirre y Francisco Armijo Castro, así como de las familias de José San Román Rouyer (principalmente su hijo, Carlos San Román Terán) y Antonio Castillo de Lucas (a través de su hijo, Antonio Castillo Ojugas), pues han aportado documentos personales y recuerdos de sus trayectorias profesionales.

La exposición se organiza en quince secciones, subdivididas algunas de ellas, que recorren los antecedentes que motivaron la necesidad de una cátedra, las circunstancias para la creación de la misma, la labor de los distintos catedráticos y profesores encargados de ella y la relación con otras disciplinas científicas.

En el presente catálogo se detallan todos los documentos exhibidos, tal y como se pueden encontrar en la exposición, más las características principales de la de tipo virtual.

\section{AGRADECIMIENTOS}

La profesora Josefina San Martín Bacaicoa, catedrática emérita de la disciplina, ha querido que la Cátedra de Hidrología Médica de la Facultad de Medicina de la Universidad Complutense, al cumplir sus cien años (1912-2012), tuviese el adecuado reconocimiento y homenaje, y que éstos quedasen reflejados en la vida académica de la Facultad. Han sido sus esfuerzos y su decidido entusiasmo desde el primer momento los que han propiciado la realización del proyecto. Su intención ha venido motivada no sólo por la importancia que, desde el punto de vista del afianzamiento de la disciplina, tuvieron las actividades docentes e investigadoras de sus titulares, sino también por la visibilidad que esta disciplina debería tener de cara a su futuro desarrollo y continuidad. De este modo la exposición pretende afianzar la docencia de la Hidrología Médica, profundizar en la medicina terapéutica, reforzar el Instituto de Hidrología y activar las relaciones con el mundo empresarial del agua, sector de notable peso económico en la industria balnearia y turística española.

La profesora San Martín propuso al profesor José Luis Álvarez-Sala Walther, decano de la Facultad, diferentes actos académicos en los que se celebrara la efemé- 
ride. Desde el Decanato se ha apoyado de manera decidida este programa de eventos, organizando las reuniones necesarias de todas las personas implicadas para su exitoso desarrollo. El decano, a su vez, también propuso que se planteara la iniciativa a la Dirección de la Biblioteca para que se concretase en una exposición que recogiera los trabajos y el desarrollo de la disciplina durante estos cien años.

Desde la Biblioteca de la Facultad se han plasmado dos iniciativas: una exposición física, bibliográfica, que permaneciera abierta durante un amplio periodo de tiempo (hasta finales del mes de junio de 2012) y otra virtual. Su objetivo ha sido que difundieran de manera extensa y permanente la historia de la enseñanza de la Hidrología Médica y el estado de la cuestión en el momento presente.

La realización de ambas exposiciones, no habría podido efectuarse sin el minucioso y esforzado trabajo de todo el personal de la Biblioteca de la Facultad en la preparación y realización de la exposición virtual de la que disfrutamos en la web. Del mismo modo, se ha promovido la necesaria concurrencia y asistencia de profesores e investigadores vinculados a la disciplina con el fin de colaborar en la definición del itinerario científico, así como para obtener el necesario patrocinio. En este sentido hay que señalar la labor de la doctora San Martín para implicar, en mayor o menor medida, a los balnearios de Sicilia y de Serón, Balneario El Raposo y el Balneario de Lugo.

En esta línea de coordinación también se ha vinculado al Departamento de Medicina Física y de Rehabilitación. Hidrología Médica, en la persona del doctor Antonio Álvarez Badillo, jefe de dicho departamento. Asimismo, dado que España acogería -en Lanjarón-Granada, durante el mes de junio de 2012- el $38^{\circ}$ Congreso de la Sociedad Internacional de Hidrología Médica y Climatología (I.S.M.H.) Medical Hydrology and Balneology: Environmental Aspects, los profesores Francisco Maraver Eyzaguirre, director de la Escuela de Hidrología Médica de esta facultad, y Francisco Armijo de Castro se han sumado decididamente a la propuesta inicial con el objetivo de aunar esfuerzos, establecer vínculos entre los actos del $38^{\circ}$ Congreso y aumentar la proyección y difusión de dicho congreso en el ámbito estatal e internacional. Ambos han ayudado a ultimar la preparación de las exposiciones, aportando instrumental y documentación institucional y personal. La clausura de la exposición ha coincidido con un acto académico y la visita de los ponentes y asistentes al $38^{\circ}$ Congreso, en la Facultad el día 19 de junio.

Los fondos expuestos provienen en su mayor parte de la Biblioteca de la Facultad de Medicina pero también se han expuesto otros fondos de las Bibliotecas de las facultades de Derecho, Farmacia, Geológicas, Odontología y Veterinaria, cuyos directores han prestado su colaboración en todo momento.

Sin la tarea de búsqueda, selección y expurgo que José Luis Gutiérrez Menéndez realiza en el Archivo de la Facultad de Medicina, no hubiéramos dispuesto de unos documentos de gran valía para historiar las vicisitudes administrativas de la cátedra, que han sido presentados en la exposición y escaneados para su exhibición virtual. 
Un agradecimiento especial merece la Biblioteca Histórica de la Universidad Complutense de Madrid "Marqués de Valdecilla", que ha prestado varias obras de su fondo pertenecientes a los siglos XVI, XVII, XVIII y XIX. Marta Torres Santo Domingo, su directora, y Aurora Díez Baños, jefe de Procesos e Información Especializada, han organizado los fondos cedidos. El Departamento de Conservación y Restauración dirigido por Javier Tacón Clavaín, reconocido especialista en el tratamiento y conservación de fondos bibliográficos y documentales, ha dispuesto las medidas de traslado y conservación de los fondos en las vitrinas expositoras.

Estas vitrinas, realizadas en nogal español en el siglo XIX, pertenecen al mobiliario antiguo del patrimonio complutense y han sido limpiadas, renovado su sistema de iluminación y restauradas por el servicio de mantenimiento y conservación de la Facultad.

La Facultad de Medicina, a través de su Gerencia, ha posibilitado la instalación y custodia de la exposición en la Sala de Profesores, contigua a la Sala de Juntas "Profesor Nogales", en la segunda planta de dicha facultad. El gerente D. Jesús Bragado, ha dispuesto los recursos económicos, el personal de obras y servicios y cuantos otros recursos han resultado imprescindibles para la instalación de la exposición. El técnico especialista D. Ángel Luis Jorge, responsable de los servicios de la facultad, junto con el personal a su cargo han realizado una encomiable labor facilitando en todo momento la instalación, limpieza, custodia y atención de la exposición.

Destacar, por último, la tarea de difusión de los actos académicos y de la exposición en todo el ámbito médico profesional e institucional llevada a cabo por la Dra. María Jesús Pascual Segovia, médico, especialista en Medicina Preventiva y Salud Pública, quien actualmente desempeña su actividad en la Unidad de Comunicación Madrid Salud del Ayuntamiento de Madrid.

Del mismo modo, desde el Gabinete de Comunicación de la Universidad Complutense, Isaac García del Río, director de Comunicación, y Pilar Martín han prestado la máxima difusión a los actos y a la exposición, dándoles una gran preeminencia informativa en la web de la universidad y en la revista digital Tribuna Complutense.

\section{Los antecedentes: primeros tratadistas médicos}

La utilidad de las aguas mineromedicinales como efectivo recurso terapéutico llevó a que fuesen sumamente estimadas incluso desde épocas prehistóricas. En España, el intento de unificación territorial bajo el reinado de Isabel I y Fernando V, auspició la elaboración de una imagen nacional a través del inventario de sus grandezas y los manantiales mineromedicinales se encontraron entre ellas. El pensamiento científico del siglo XVII, de la alquimia a la iatroquímica, buscó comprender el efecto de las aguas mediante rudimentarios análisis. El tratamiento hidromineral, aunque seguiría coexistiendo con lo popular y creencial, comenzó a 
ser reivindicado desde la ciencia médica. Si la obra de Alfonso Limón Montero es considerada la primera gran recopilación de manantiales españoles, la inconclusa de Bedoya y, sobre todo, la de Juan de Dios Ayuda supusieron la introducción sistemática del análisis químico contemporáneo de Bergman y Fourcroy.

Cura de la piedra y dolor de la ijada / Julián Gutiérrez de Toledo

Toledo: Pedro Hagenbach; a expens. de Melchor Gorricio, 4 abril, 1498. (ed. Facsímil)

Incunable 1 v.: il.; Fol.

BH INC M-29

Biblioteca Histórica. UCM

Figura 1 - Cura de la piedra y dolor de la ijada

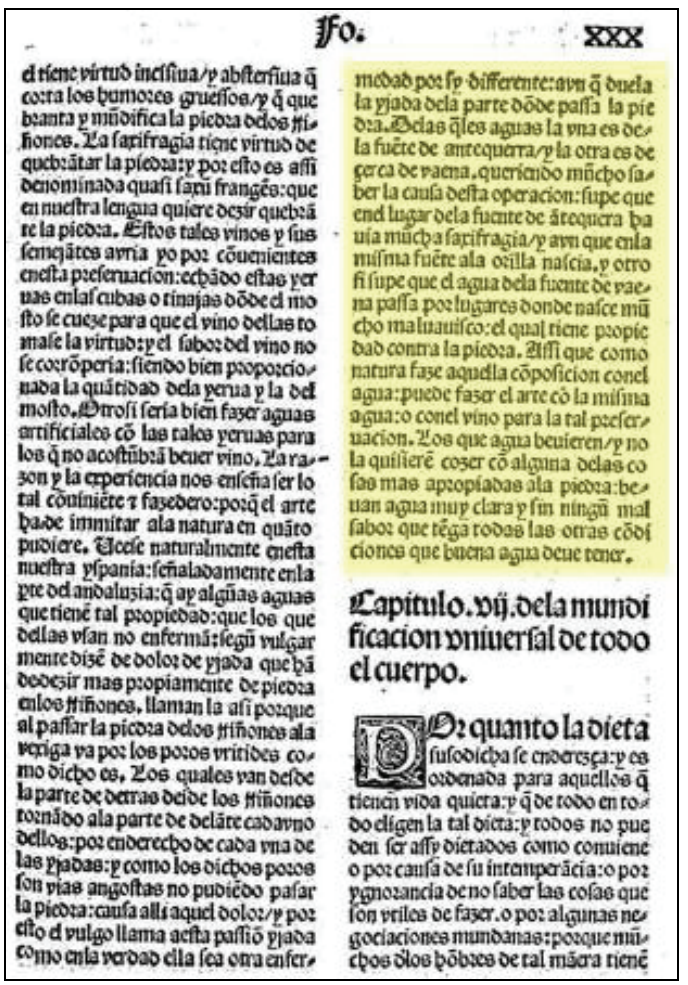

Historia universal de las fuentes minerales de España, sitios en que se hallan... : tomo primero que comprehende las letras A y B / su autor D. Pedro Gomez de Bedoya y Paredes... 
En Santiago de Compostela: en la imprenta de Ignacio Aguayo, [1764]

[8], 435, [2] p., [2] en bl., [1] h. de grab. ; $4^{\circ}(21 \mathrm{~cm})$

Sign.: BH FOA 5024

Biblioteca Histórica. UCM

Examen de las aguas medicinales de mas nombre que hay en las Andalucias: en que se da noticia de la situacion, contenidos, virtudes y metodo con que deben usarse las de cada fuente / por D. Juan de Dios Ayuda; tomo I, contiene los Baños de Graena, Alicun, y Baza

En Baeza: por D. Agustín de Doblas, impresor de la Universidad, 1793

XXXIX, 278 p., [2] en bl. ; $8^{\circ}$

Sign.: BH FOA 4345

Biblioteca Histórica. UCM

\section{La obra del Cuerpo de Médicos de Baños (1816-1877)}

En 1816, un Real Decreto de Fernando VII, creaba el Cuerpo de Médicos de Baños, con funciones administrativas, investigadoras y clínicas en los más concurridos balnearios españoles. Percibidos bien pronto como una élite dentro de la clase médica (pues contaron hasta 1868 con sueldo del Estado y retribución por cada bañista atendido), esta corporación desarrolló una intensa labor para transformar la práctica balnearia en ciencia hidrológica. Aunque con unos conocimientos analíticos y clínicos demostrados en oposición, la obligación de presentar una topografía médica de la localidad balnearia les llevó a desarrollar una formación autodidacta en las materias más diversas. Pedro María Rubio pudo dar unidad en su obra a estos esfuerzos dispersos durante más de tres décadas.

Opúsculo en que se manifiestan los beneficios que reportan la salud pública y la riqueza nacional, de que las Cortes y el Gobierno protejan con energía el importante y utilísimo ramo de aguas minerales / González Crespo, Mariano José Madrid: Imp. León Amarita, 1838

$31 \mathrm{p}$; $18 \mathrm{~cm}$.

Sign.: Fo 1357(10)

Biblioteca Facultad de Medicina. UCM

Tratado completo de las fuentes minerales de España / por Pedro María Rubio Madrid: Est. Tip. de R.R. de Rivera, 1853

XXXII p. ; $23 \mathrm{~cm}$

Sign.: DA 6811

Biblioteca Facultad de Medicina. UCM 
Figura 2 - Portada

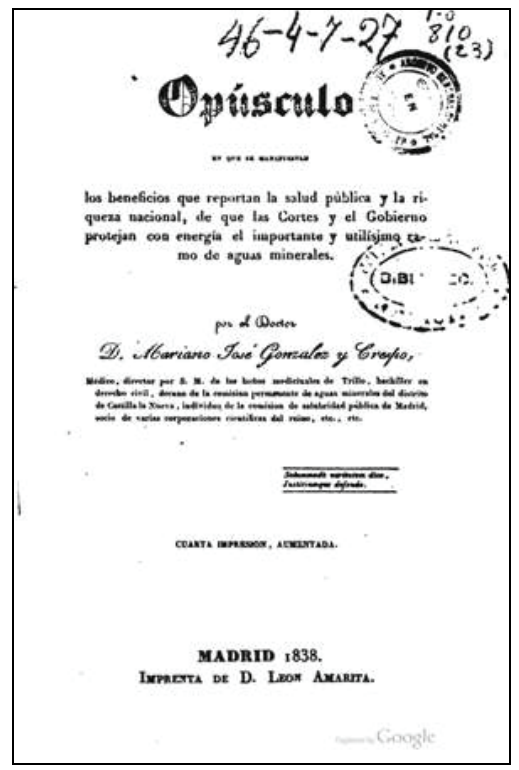

Manual de las aguas minerales de España y principales del extranjero / por Francisco Alvarez Alcalá

Madrid y Santiago: Librerías de Don Angel Calleja; Lima: Casa de los Señores Calleja, Ojea y Comp., 1850 (Imp. de Don Alejandro Gomez Fuentenebro)

256 p. ; $20 \mathrm{~cm}$

Sign.: DA 6822

Biblioteca Facultad de Medicina. UCM

\section{La Sociedad Española de Hidrología Médica: el proceso de institucionali- zación de la disciplina}

En 1877 la constitución del escalafón de antigüedad del Cuerpo de Médicos de Baños ponía de manifiesto las diferencias entre sus miembros. En un intento de dar cohesión científica y social a este colectivo surgió pocos días después la Sociedad Española de Hidrología Médica. La Sociedad supuso una breve época de esplendor científico por su promoción de la investigación y la docencia, con la creación de un laboratorio químico para análisis de aguas y la publicación de su revista oficial, los Anales, cuyos artículos ejemplificaban la calidad en este tipo de estudios. Junto al Anuario Oficial de las Aguas Minerales de España ofrecieron el mejor conocimiento de las aguas y balnearios de España y exhibieron el nivel internacional alcanzado por la ciencia hidrológica, si bien sólo por un reducido grupo de hidrólogos. Las 
desigualdades inherentes a un escalafón de antigüedad minaron el proyecto con una desaparición temporal de las publicaciones y la aparición de la Revista MédicoHidrológica Española, considerada por algunos coetáneos como "la izquierda del escalafón".

Censo general de las aguas minerales de España / por D. Mariano Carretero y Muriel...

Madrid: Imp. de F. García y D. Caravera, 1876

57 p., [1] h.: tab. ; $21 \mathrm{~cm}$

Sign.: Ca 2861(5)

Biblioteca Facultad de Medicina. UCM

Figura 3 - Portada

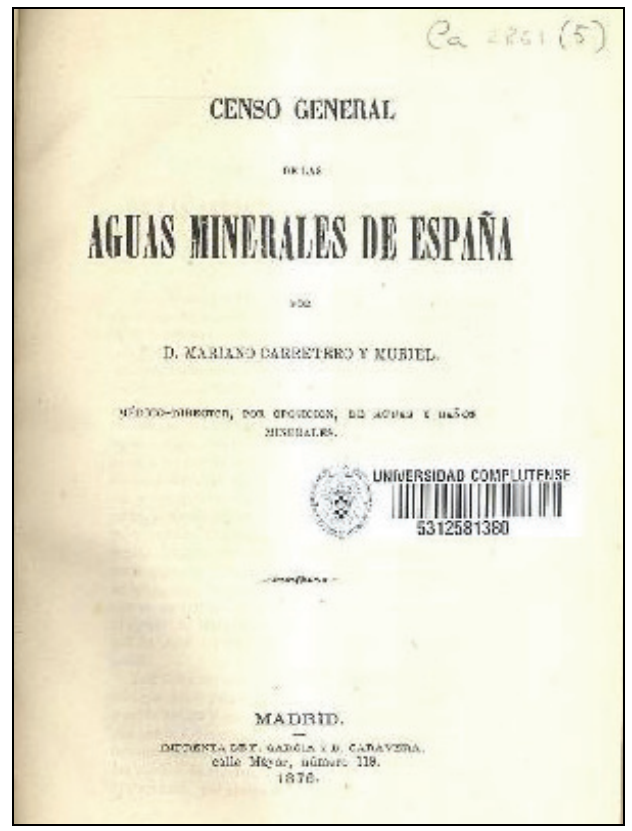

Anuario oficial de las aguas minerales de España

Madrid: Manuel Minuesa de los Rios, 1876-1890

Sign.: PP0MED A57

Biblioteca Facultad de Medicina. UCM 
Resumen estadístico oficial de las aguas minerales de España / Marcial Taboada y Mariano Carretero y Muriel

Madrid: Imprenta de Ricardo Rojas, 1890-1900

Sign.: PP0MED A149

Biblioteca Facultad de Medicina. UCM

Colección legislativa de baños y aguas minero-medicinales / por Carlos Menéndez y Joaquín María Aleixandre; con una carta prólogo de Marcial Taboada

Madrid : Imp. de Ricardo Rojas, 1892

VII, 346 p. ; $18 \mathrm{~cm}$

Sign.: DA 6739

Biblioteca Facultad de Medicina. UCM

Reseña de los principales balnearios de España: libro dedicado a los miembros del XIV Congreso Internacional de Medicina, abril, 1903 / por los médicos directores de baños

Madrid : Imprenta de Ricardo Rojas, 1903

331 p. ; $24 \mathrm{~cm}$

Sign.: DE615.838(460)RES

Biblioteca Facultad de Medicina. UCM

Discursos leídos en la sesión de aniversario celebrada por la Sociedad Española de Hidrología Médica el día 4 de marzo de 1883/ por Benigno Villafranca y Alfaro y por José María Bonilla y Carrasco

Madrid: Establecimiento Tipográficos de M. Minuesa, 1883

54 p., [1] h. ; $26 \mathrm{~cm}$

Sign.: Fo 1241(11)

Biblioteca Facultad de Medicina. UCM

Anales de la Sociedad Española de Hidrología Médica

Madrid: Sociedad Española de Hidrología Médica, 1877-1936

Sign.: PP0MED A14

Biblioteca Facultad de Medicina. UCM

Revista médico-hidrológica española

Madrid : Imprenta de Ricardo Rojas, 1900-1904

Sign.: PP0MED A356

Biblioteca Facultad de Medicina. UCM 


\section{La legitimación por el pasado: bibliografías y memoria}

Mientras la investigación hidrológica intentaba sentar sus bases científicas y aplicar los nuevos métodos de análisis a cada manantial en uso, había que recurrir a otras formas para demostrar la utilidad del remedio. La historia sirvió a tal fin, tanto para justificar que la antigüedad en el empleo era muestra de su eficacia como para dar una visión positivista del desarrollo de la ciencia a través del aumento en el número de publicaciones. Desde presupuestos románticos el pasado sirvió para legitimar y reivindicar la ciencia hidrológica española -frente a la consolidada hidrología francesa- mediante catálogos bibliográficos entre los que destacó la monumental obra de Leopoldo Martínez Reguera.

Discursos leídos en la solemne sesión inaugural del año de 1894 de la Real Academia de Medicina / por el Marqués de Guadalerzas Discurso pronunciado ; por Mariano Carretero y Muriel ; [Estado de la hidrología médica española] Madrid: Est. Tip. de Enrique Teodoro, 1894

32,33 p. ; $28 \mathrm{~cm}$

Sign.: Fo 839(12)

Biblioteca Facultad de Medicina. UCM

Bibliografia hidrológico-médica española / por Leopoldo Martínez Reguera Madrid: Sucesores de Rivadeneyra, 1896-1897

$2 \mathrm{v}$; $28 \mathrm{~cm}$

Sign: A01"18"MR39261

Biblioteca Facultad de Farmacia. UCM

Figura 4 - Portada

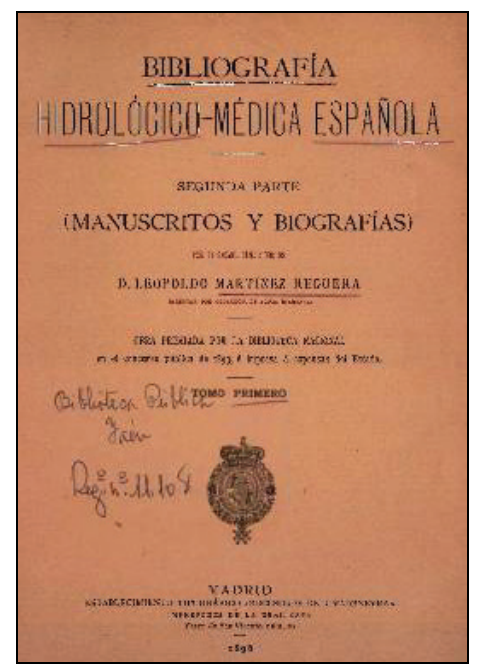


Nuevos elementos de terapeútica y de materia médica : con un ensayo sobre el arte de formular, y un análisis de aguas minerales de Francia / J. L. Alibert; trad. de la $4^{a}$ ed. por D. J. C. añadido de un análisis de las aguas minerales de España

Madrid: Imprenta de Repullés, 1826

$4 \mathrm{v} . ; 21 \mathrm{~cm}$

Sign.: BH MED 14303

Biblioteca Histórica. UCM

Materiales para una bibliografia del agua en España / reunidos y acarreados por el Conde de las Navas al pie de la obra del Excmo. Sr. D. Rafael Casset y Chinchilla

Madrid: [s.n.], 1910 (Imp. Suc. de M. Minuesa de los Rios)

$30 \mathrm{p}$.

Sign.: BH R Separatas 563

Biblioteca Histórica UCM

\section{Sistematizar conocimientos: en busca de un tratado}

El rápido desarrollo de la química durante el siglo XIX y su aplicación al análisis de las aguas mineromedicinales supusieron importantes retos en la formación de un médico de balneario si quería realizar bien su función. Los excelentes estudios, cada vez más abundantes, sobre balnearios concretos carecían de un nivel de teorización suficiente. La necesidad de contar con tratados que sistematizasen lo conocido hasta el momento en materia hidrológica llevó a que, desde mediados del siglo XIX, fuesen apareciendo publicaciones con tal pretensión. A este interés se unían las necesidades de aquellos médicos que aspiraban a aprobar las oposiciones para ingresar en el aún prestigioso Cuerpo de Médicos de Baños. Si la reconocida, premiada y reeditada obra de García López demuestra la primera finalidad, la de Aguilar Martínez confirma la segunda.

Ensayo práctico sobre la acción terapéutica de las aguas minerales : novísima recopilación de cuanto se ha escrito sobre hidrología general y especial ... ilustrada lo con el método adoptado por Henry Rose, para analizar ... / por Raimundo de Monasterio y Correa

Madrid: Imp. de D. Anselmo Santa Coloma y Comp., 1850

406 p. ; $21 \mathrm{~cm}$

Sign.: DA 6775

Biblioteca Facultad de Medicina. UCM 
Novísimo manual de hidrologia médica española ... / por José Pérez de la Flor y Manuel González de Jonte

Madrid: Imprenta y Librería de D. Vicente Matute, 1851

2 h. 658 p. 5 h. 2 h. pleg. ; $16 \mathrm{~cm}$

Sign.: DA 6770

Biblioteca Facultad de Medicina. UCM

Hidrología medica, con nociones de hidrografía, geografia, geología... /por Anastasio García López

Salamanca: Imprenta de Sebastian Cerezo, 1875

$2 \mathrm{v}$; $21 \mathrm{~cm}$

Contiene un map. pleg. de los balnearios de España al fin del t. II

Sign.: DA 6784

Biblioteca Facultad de Medicina. UCM

Figura 5 - Portada

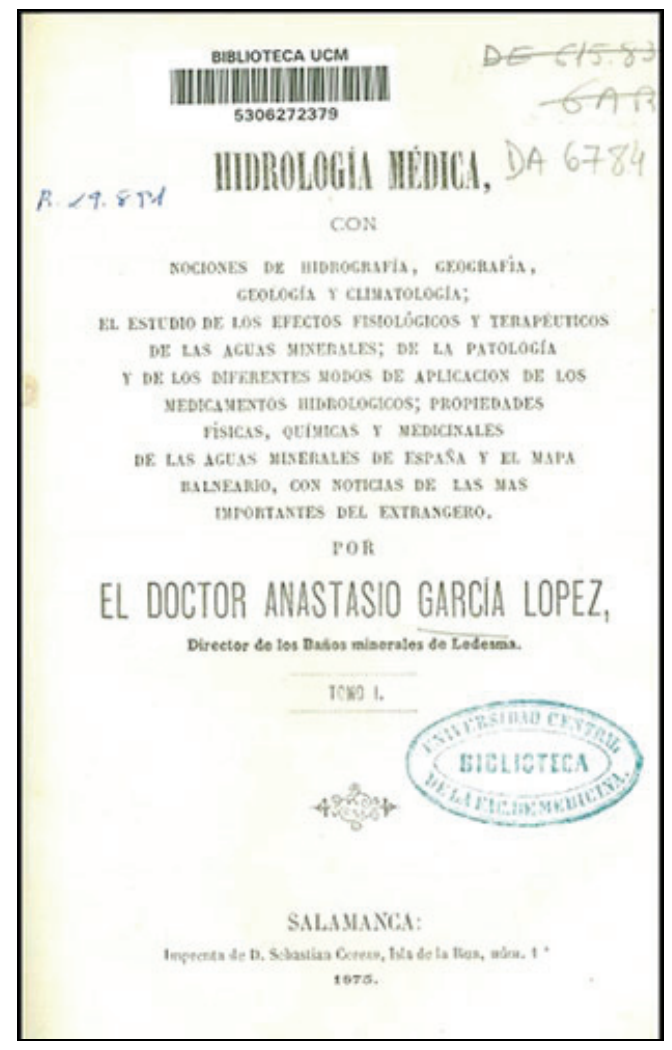


Apuntes de hidrología médica. Ideología física hidrológica según el programa de oposición para el ingreso en el cuerpo de médicos-directores de baños / por Francisco de B. Aguilar Martínez

Valencia: Imp. de Gombau, Vicent y Masiá, 1896

310 p. ; $26 \mathrm{~cm}$.

Sign.: DA 6825

Biblioteca Facultad de Medicina. UCM

\section{La industria balnearia}

Si el pasado de las aguas mineromedicinales es remoto, el del balneario en España data del siglo XIX. El balneario existe sólo desde su concepción como empresa (pública o privada) que mercantiliza el agua y los servicios necesarios para quienes la consumen. Desamortización y burguesía industrial aprovecharon y propiciaron la moda de tomar las aguas, asignando así a la ciencia hidrológica y los médicos de baños sólo una parte del complejo universo balneario. Esta pugna en el difícil equilibrio entre medicalización y desmedicalización, entre propietarios y médicos de baños, partía de una legislación que consideraba que todo balneario debía ser dirigido por un médico del Cuerpo y que eran éstos -y no los propietariosquienes podían elegir en cual desempeñar su cargo. Creada la Asociación Nacional de la Propiedad Balnearia en 1871, dedicó sus esfuerzos a defender sus intereses, pero también a dar publicidad a sus establecimientos, sin dudar en incorporar todas las aportaciones que los médicos iban descubriendo sobre las virtudes de sus aguas.

La libertad balnearia : contienda entre propietarios y médicos-directores de balnearios / publicada por la Junta Directiva de la Asociación de Propietarios de Balnearios y Manantiales de Aguas Minero-Medicinales de España

Madrid: Est. Tip. de Jaime Ratés, 1910

227 p. ; $20 \mathrm{~cm}$

Sign.: DE615.838(460)LIB

Biblioteca Facultad de Medicina. UCM

Guía-manual de baños y aguas minerales destinada al servicio del público... : ampliada con la legislación de aguas en general y otras curiosidades / por [Saturnino de Arce y Cortázar]

Madrid: Imprenta Nacional, 1873

476 p. ; $17 \mathrm{~cm}$

Sign.: FH 2410

Biblioteca Facultad de Educación. UCM 
Figura 6 - Cubierta

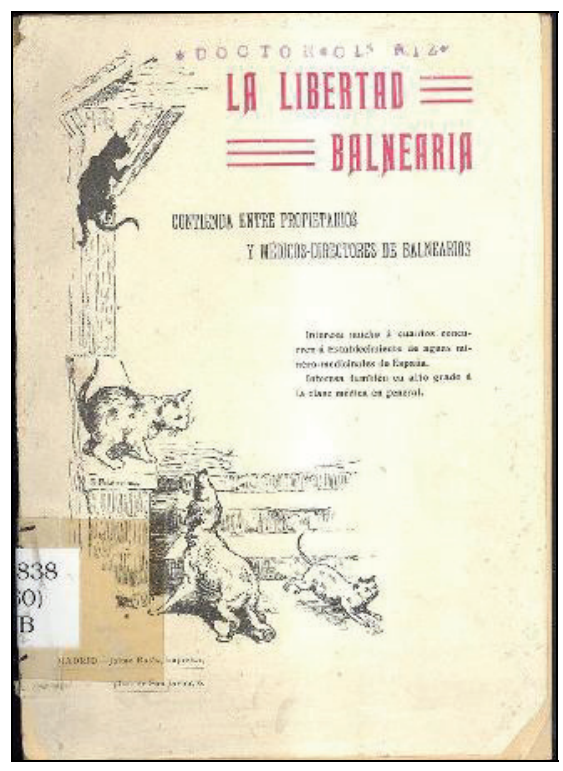

Guía ilustrada de las aguas minerales y balnearios de España : obra de gran utilidad para todo el que necesite usar aguas minerales y acudir á los balnearios / colaboración de distinguidos Médicos ... bajo la dirección de D. Ricardo de la Puerta y Escolar ... ; con grabados intercalados en el texto Madrid: [s.n.], 896 [i.e. 1896]( Imprenta Colonial, á cargo de G. Gutierrez) 135, [9] p. : il. ; $22 \mathrm{~cm}$

Sign.: Fo 1326(5)

Biblioteca Facultad de Medicina. UCM

Guía de los establecimientos balnearios de España : temporada de 1897 / Miguel Dávila

Madrid: Administración, [1897?]

390 p. ; 8 a

Sign.: DE615.838DAV

Biblioteca Facultad de Medicina. UCM

Reseña de los principales balnearios de España: libro dedicado a los miembros del XIV Congreso Internacional de Medicina, abril, 1903 / por los médicos directores de baños

Madrid: Imprenta de Ricardo Rojas, 1903

331 p. ; c $24 \mathrm{~cm}$

Tít. de la cub.: Aguas minero-medicinales de España 
Sign.: DE 615.838(460) RES

Facultad de Medicina. UCM

Guía oficial de las aguas minero-medicinales y establecimientos balnearios de España

Madrid: Est. Tip. de Valentín Tordesillas, 1897-1947

Sign.: PPOMED A19

Biblioteca Facultad de Medicina. UCM

Guía oficial de los establecimientos balnearios y aguas medicinales de España: año 1927 (R.O. de 28 agosto 1926)

Barcelona : Rudolf Mosse, 1927

358 p. : il. ; $24 \mathrm{~cm}$

Sign.: F.A./281

Biblioteca Facultad de Odontología. UCM

Guía de los establecimientos-balnearios de aguas minero-medicinales en España / Luis Marty-Herráez

Madrid: [s.n.], 1942

1 v. (pág. var.) ; $23 \times 32 \mathrm{~cm}$

Sign.: DE615.838(460)MAR

Biblioteca Facultad de Medicina. UCM

\section{La guía del bañista}

Los agüistas son, junto con el agua, la esencia del balneario. Desde el primer Reglamento de Baños de 1817 los médicos tuvieron que cumplimentar estadísticas de concurrencia y de resultados terapéuticos, en las que también se hacía constar si los pacientes eran militares, acomodados o pobres de solemnidad. Estos últimos podían llegar a una importante proporción en algunas regiones y balnearios y acudían con el viaje pagado por sus ayuntamientos. Los más numerosos, no obstante, eran los acomodados, si bien esta categoría incluía desde los más acaudalados visitantes de los más afamados balnearios a los modestos concurrentes de establecimientos familiares. Para todos ellos las guías del bañista supusieron una información para la terapéutica y el ocio con tinte publicitario que anticipaban ofertas de turismo de salud.

Guía para los baños de Cestona / por su médico director Justo Ma Zavala Azpeitia : Imp. de P. Martínez, 1868

1 v. pag. var. ; $21 \mathrm{~cm}$

Sign.: Ca 2861(3)

Biblioteca Facultad de Medicina. UCM 
Vademecum-guía del médico y del bañista de los balnearios de aguas minerales de España: su itinerario, situación, clase, virtudes medicinales, temporada oficial, instalacion, [etc.]..., / por la redacción del periódico médico-farmacéutico Los Avisos que dirige Pablo Fernández Izquierdo y redacta Federico Gómez de la Mata Madrid: Los Avisos, 1885

189 p. ; $21 \mathrm{~cm}$

Sign.: Ca 2861B(10)

Biblioteca Facultad de Medicina. UCM

Baños y aguas minerales de Archena (Provincia de Murcia) : [guía]

Madrid: E. Teodoro, 1886

1 folleto pleg. de 12 p. ; $16 \mathrm{~cm}$

Sign.: Ca 2735(11)

Biblioteca Facultad de Medicina. UCM

Guía del balneario de Santa Teresa de Ávila... / por Domingo Fernández Campa... Ávila: Tip. de Cayetano González, 1897

37 p., [1] h. ; $\mathrm{c} 22 \mathrm{~cm}$. Memoria manuscrita y folleto impreso

Acompaña manuscrito con la memoria y cuadros estadísticos y folleto impreso:

“Análisis químico y micrográfico cualitativo y cuantitativo...", Ávila, 1896

Sign.: Ca 2837(19-2)

Biblioteca Facultad de Medicina. UCM

Figura 7 - Cubierta

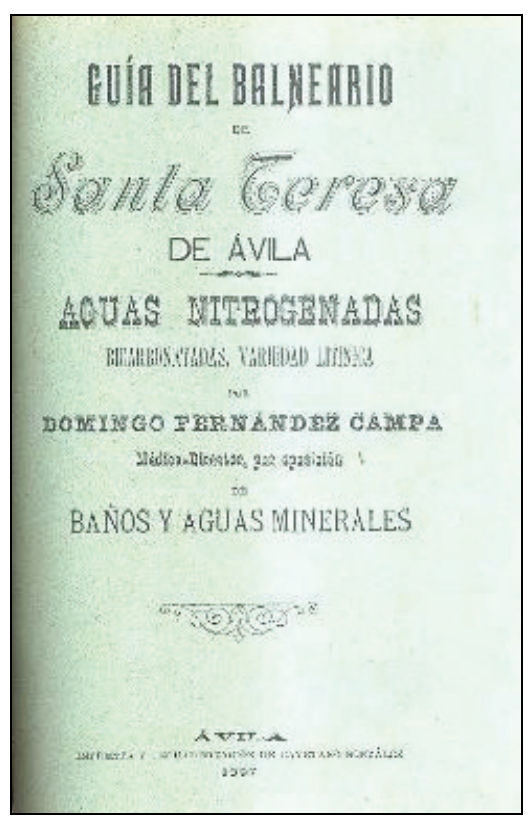


Indicador terapeútico y guía del bañista en Betelu (Navarra)... [Microforma] / médico director en propiedad Dr. D. Manuel Millaruelo

Zaragoza : Imp. de Comas, 1899

31 p. ; $20 \mathrm{~cm}+$ memoria manuscrita

Sign.: Navarra(II) Betelú(23)

Biblioteca Facultad de Medicina. UCM

\section{La creación de la Cátedra de Hidrología Médica}

Los médicos adquirían conocimientos sobre las aguas mineromedicinales de forma autodidacta y a través de los breves temas de la asignatura de "Materia Médica". Desde el Cuerpo y la Sociedad se reclamaba la necesidad de que los estudios de la disciplina quedasen institucionalizados a través de la creación de una cátedra universitaria específica. Cuando Amalio Gimeno (médico director del balneario de Cestona) ocupó el Ministerio de Instrucción Pública hizo realidad esa pretensión, considerando que contribuiría a revitalizar los conflictivos órganos oficiales de la disciplina. Un Real Decreto de 5 de enero de 1912 creaba la Cátedra de Hidrología Médica como de libre elección entre las del doctorado. El resultado de la oposición dio la plaza en 1913 a Hipólito Rodríguez Pinilla, quien desarrolló una intensa labor de difusión de los estudios hidrológicos, potenció la investigación, defendió la creación de un Instituto de Hidrología y Climatología Médicas y creó en torno a él una escuela hidrológica (con José García Viñals y Antonia Martínez Casado). Miembro de la Real Academia Nacional de Medicina y Consejero de Estado su actividad y propuestas cambiaron la disciplina y condujeron a una controvertida y breve supresión del Cuerpo en aras de la creación de la especialidad.

\subsection{La enseñanza de la Hidrología Médica antes de la cátedra}

Elementos de terapéutica y materia médica / por el doctor Ramon Capdevila Madrid : Imprenta de Leon Amarita, 1825 202 [i.e. 102], 300 p. ; $16 \mathrm{~cm}$

Sign.: BH MED 14753

Biblioteca Histórica. UCM

Noticia de las aguas minerales más principales de España : apéndice al curso de materia médica o farmacológica / por Juan Bautista Foix y Gual

Barcelona: por Joaquín Verdaguer, 1840

138 p. ; $17 \mathrm{~cm}$

Sign.: DA 12844

Biblioteca Facultad de Medicina. UCM 
Programa de ampliación de la terapéutica é hidrología médica / por Vicente Asuero y Cortazar

Madrid: Imprenta Médica de M. Alvarez, 1868

LXXXVII ; $20 \mathrm{~cm}$

Sign.: DA 7436

Biblioteca Facultad de Medicina. UCM

Curso elemental de terapéutica, materia médica y arte de recetar con nociones de hidrología médica para uso de los alumnos de tercer año / por Vicente Peset y

Cervera

Valencia: Imprenta de Federico Domenech, 1894

2 v. ; $22 \mathrm{~cm}$

Sign.: DA 7313-14

Biblioteca Facultad de Medicina. UCM

Oficio del decano de la Facultad de Medicina, Jesús Calleja, dirigido al Excmo. Señor Ministro de Instrucción Pública y Bellas Artes en el que da cuenta de la creación de una Cátedra de Hidrología Médica.

2 de julio de 1911

Archivo de la Facultad de Medicina. UCM

Estudios de hidrología médica / por el Dr. Rodríguez Pinilla

Madrid: Imprenta de Ricardo F. de Rojas, 1914

60 p. ; $16 \mathrm{~cm}$.

Sign.: Fo 823(1)

Biblioteca Facultad de Medicina. UCM

Figura 8 - Cubierta

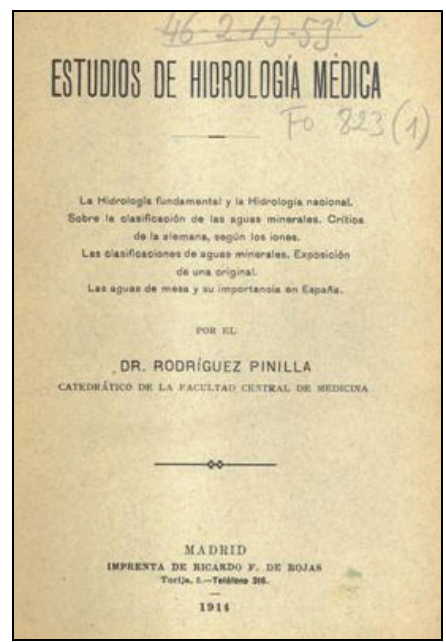




\subsection{Hipólito Rodríguez Pinilla: la creación del corpus teórico hidrológico}

Elementos de terapéutica y materia médica / por el doctor Ramon Capdevila Madrid: Imprenta de Leon Amarita, 1825 202 [i.e. 102], 300 p. ; $16 \mathrm{~cm}$

Sign.: BH MED 14753

Biblioteca Histórica. UCM

Estudios de hidrología médica / por el Dr. Rodríguez Pinilla

Madrid: Imprenta de Ricardo F. de Rojas, 1914

60 p. ; $16 \mathrm{~cm}$.

Sign.: Fo 823(1)

Biblioteca Facultad de Medicina. UCM

Diccionario general hidrológico comprendiendo términos de físico-química y geología / por H. Rodriguez Pinilla

Madrid: Imp. y Encuadernación de Julio Cosano, 1916

408 p. ; $22 \mathrm{~cm}+1$ h. de plan.pleg.

Sign.: DE616.838(03)ROD

Biblioteca Facultad de Medicina. UCM

Medicaciones hidrológicas : (en cinco mapas) / Hipólito Rodríguez Pinilla

Madrid: Saturnino Calleja, cop. 1920

126 p. ; $16 \mathrm{~cm}$.

Sign.: DE615.838ROD

Biblioteca Facultad de Medicina. UCM

Clínica hidrológica española : con un estudio fisico-quimico de las aguas minerales / por E. Doz, Hipólito Rodríguez Pinilla ... [et al.] ; prefacio de Amalio Gimeno Madrid: Calpe, 1922

XV, 528 p. ; $21 \mathrm{~cm}$

Sign.: DE615.838CLI

Biblioteca Facultad de Medicina. UCM

Manual de hidrología médica / por H. Rodríguez Pinilla

Madrid: Reus, 1925

377 p. ; $16 \mathrm{~cm}$.

Sign.: DE615.838ROD

Biblioteca Facultad de Medicina. UCM 
Figura 9 - Cubierta

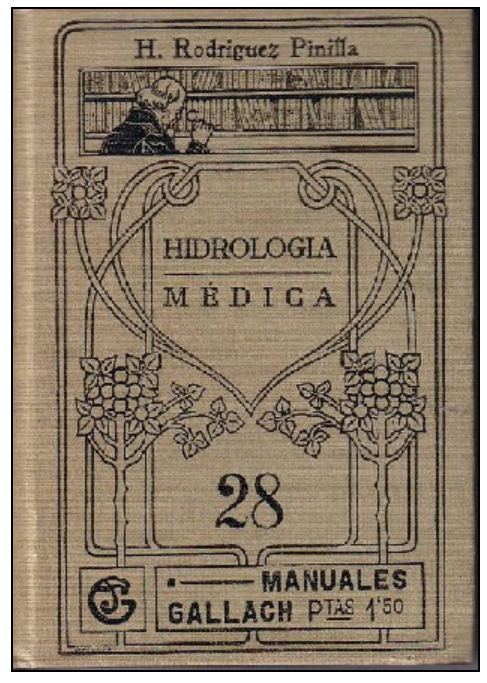

Oficio del Ilmo. Sr. Director General de Enseñanza Superior y Secundaria dirigido al Ilmo. Sr. Rector de la Universidad de Madrid, en el que hace disponer que la cátedra de "Hidrología médica" se denomine en lo sucesivo "Hidrología y climatología médicas".

12 de agosto de 1926

Archivo de la Facultad de Medicina. UCM

Manual de climatología general y española / por H. Rodríguez Pinilla Madrid: J. Cosano, 1930

242 p. ; $22 \mathrm{~cm}$

Sign.: DE551.58ROD

Biblioteca Facultad de Medicina. UCM

\subsection{Hipólito Rodríguez Pinilla, médico de baños}

Balneario de Arteijo (La Coruña)

Memoria clínica-terapéutica y administrativa del establecimiento balneario de Arteijo en la temporada de 1888 / [director] Hipólito Rodríguez Pinilla. 1888

Sign.: Depósito La Coruña Arteijo(22)

Biblioteca Facultad de Medicina. UCM 
Figura 10 - Cubierta

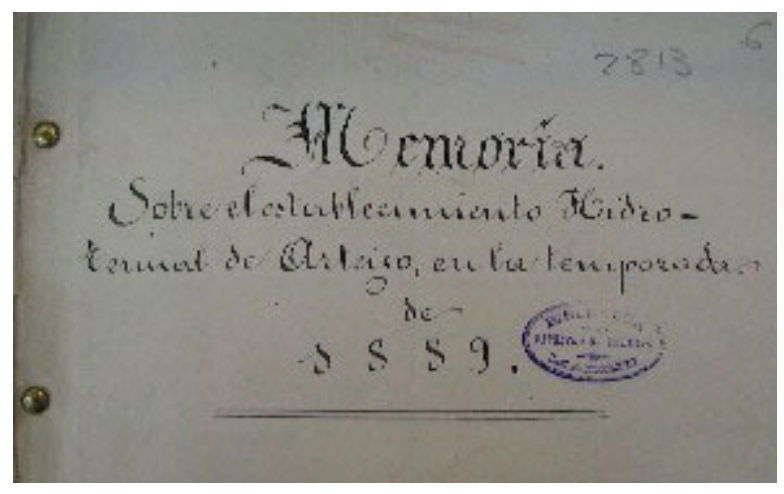

Memoria sobre el establecimiento hidro-termal de Arteijo en la temporada de 1889 / [director] Hipólito Rodríguez Pinilla. 1889

[8] h. : tabl. ; $15 \mathrm{~cm}$

Incluye cuadros estadísticos

Sign.: La Coruña-Arteijo(26)

Biblioteca Facultad de Medicina. UCM

Balneario de Caldas de Reyes (Pontevedra)

Balneario de Caldas de Reyes (provincia de Pontevedra) : memoria clínica en la temporada oficial de 1890 / [director] H. Rodríguez Pinilla, 1890

Sign.: Depósito Pontevedra (III) Caldas Reyes (24)

Biblioteca Facultad de Medicina. UCM

Aguas del Incio(en Lugo) : memoria de la temporada de 1894 / H.Rodriguez Pinilla [10] h.; $22 \mathrm{~cm}$

Incluye cuadros estadísticos

Sign.: Lugo(I) Incio(1)

Biblioteca Facultad de Medicina. UCM 


\subsection{Los primeros discípulos: José García Viñals}

Tratamiento de las enfermedades del aparato respiratorio por las aguas sulfuradas-cálcicas, frías, sulfhídrico-nitrogenadas / por José García Viñals Tesis de la Universidad Central (Madrid), Facultad de Medicina, 1916

Sign.:Fo 2059(4)

Biblioteca Facultad de Medicina. UCM

Tratamiento de las enfermedades de la infancia por las aguas minero-medicinales españolas / por Carlos S. de los Terreros y José García Viñals ; con un prólogo .. H. Rodríguez Pinilla

Madrid: Establecimiento Tipográfico de Jaime Ratés, 1916

126 p. ; $17 \mathrm{~cm}$

Sign.: Fo 898(17)

Biblioteca Facultad de Medicina. UCM

Figura 11 - Portada

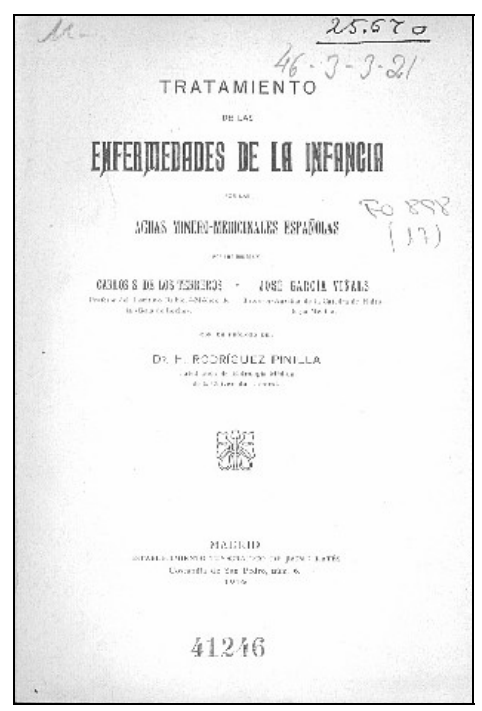

"Las aguas alcalinas de España" / por José García Viñals

En: Archivos españoles de enfermedades del aparato digestivo y de la nutrición, 1922 , t. 5, p. 651-661

Sign.: PP 0MED 1922, Vol. 5

Biblioteca Facultad de Medicina. UCM 


\section{Guerra, depuración y primer franquismo}

Cuando Rodríguez Pinilla se jubiló en 1931 quedó como profesora encargada de la cátedra Antonia Martínez Casado. La supresión temporal del Cuerpo, la desaparición de los Anales y la Guerra Civil encadenaron serios reveses para la hidrología y una consecuente decadencia. La vulnerabilidad de la cátedra propició el intento de absorción por la Terapéutica Física (en 1939) y la necesidad de apoyo en otras especialidades. La depuración de Martínez Casado y la incorporación de José de San Román propiciaron la continuidad y la oposición de 1941 a la cátedra, en que la plaza quedó vacante. Dos años más tarde se creaba una Junta Asesora de Balnearios y Aguas Minero-Medicinales en la que se diversificaba la representatividad, se clasificaban los balnearios según especialidad terapéutica y se creaba el cuerpo facultativo de Médicos de Aguas Minero-Medicinales Inspectores de Establecimientos Balnearios. Éstos últimos nutrieron a la Sociedad y la convirtieron en un órgano hostil a la cátedra, según reflejó el secretario de aquella, Juan de Dios García Ayuso, desde las páginas del Boletín Español de Hidrología Médica y Climatología.

\subsection{El contexto balneario: cambios sociales, cambios administrativos}

Junta Asesora de Balnearios y Aguas Minero-medicinales

Boletín Oficial del Estado [BOE], 1943, pp. 8323-4.

En la p. 10530 figura la primera composición de esa Junta

\subsection{Vicisitudes de la Cátedra}

Manual de prácticas hidrológicas / por Antonia M. Casado y Arturo Cervignon Díaz; con un prólogo de Hipólito R. Pinilla

Madrid: Editorial España, 1931

206 p. ; $19 \mathrm{~cm}$

Sign.: DE615.838CAS

Biblioteca Facultad de Medicina. UCM

Propuesta de una reforma parcial y provisional de Estudios de la Facultad de Medicina de Madrid en la que se propone quede suprimida la matrícula y la enseñanza de la asignatura de Hidrología médica...

29 de septiembre de 1939

Archivo de la Facultad de Medicina. UCM 
Minuta del Decano de la Facultad de Medicina al Excmo. Sr. [Rector de la Universidad Central] en la que se hacen constar las erratas publicadas en el B.O. del Ministerio de Educación Nacional. Se adjunta una relación de las cátedras existentes en la Facultad de Medicina y que deben ser dotadas convenientemente.

19 de febrero de 1942

Archivo de la Facultad de Medicina. UCM

\subsection{Entre la SEHM y la Cátedra}

Tratado completo de hidrología médica, climatología y legislación balnearia : obra de utilidad práctica para médicos hidrólogos / J. García Ayuso ; prólogo del Doctor Velasco Pajares

Madrid: Instituto Editorial Reus, 1942

463 p., 2 h. ; $22 \mathrm{~cm}$

Sign.: DE615.838GAR

Biblioteca Facultad de Medicina. UCM

Complementos de hidrología médica : (nociones de química fisiológica, metabolismos y procesos metabólicos, acciones biológicas de las aguas minerales) / Doctor

García Ayuso

Madrid : Instituto Editorial Reus, 1944

163 p., 2 h. ; $22 \mathrm{~cm}$

Sign.: D615.838GAR

Biblioteca Facultad de Farmacia. UCM

Figura 12 - Portada

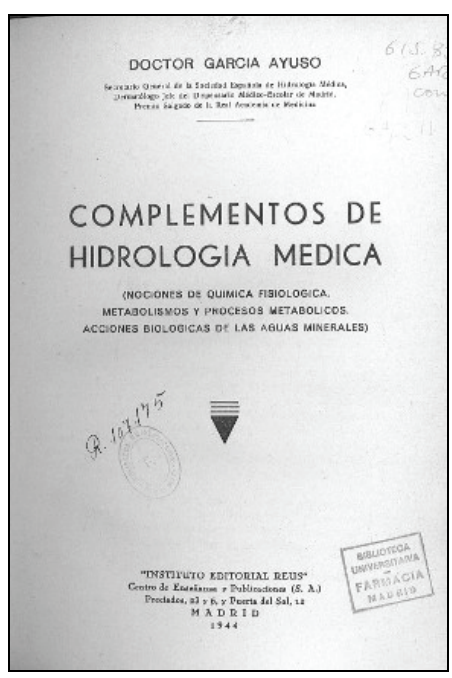


Oficio del Ilmo. Sr. Director General de Enseñanza Universitaria dirigido al Ilmo. Sr. Decano de la Facultad de Medicina, en el que se comunica que se ha resuelto que .... de dichas dotaciones se aplique una a dotar la Cátedra de "Hidrología Médica" de la Facultad de Medicina de la Universidad de Madrid.

14 de agosto de 1945

Archivo Facultad de Medicina. UCM

lo Congresso luso-espanhol de hidrología : Actas-alocuções-comunicações, 1947 [Lisboa] : Ministério da Economia, 1947

513 p. ; $30 \mathrm{~cm}$

Sign.: DE615.838(063)CON

Biblioteca Facultad de Medicina. UCM

La terapeútica hidromineral / J. García Ayuso

Madrid: Dirección General de Sanidad, 1959

104 p. ; $19 \mathrm{~cm}$

Sign.: Fo 223(8)

Biblioteca Facultad de Medicina. UCM

Crenoclimatología en España / Enrique Conde Gargollo

Madrid: Dirección General de Sanidad, 1959

84 p. ; $19 \mathrm{~cm}$

Sign.: Fo 1420(5)

Biblioteca Facultad de Medicina. UCM

Hidrología y climatología médicas / J. García Ayuso

Madrid: Cultura Clásica y Moderna, 1962

584 p. ; $22 \mathrm{~cm}$

Sign.: DE615.838GAR

Biblioteca Facultad de Medicina. UCM

\section{José San Román y el "Instituto Alfonso Limón Montero"}

Incorporado en 1939 como profesor auxiliar, opositó a la cátedra en 1941. Tras esa convocatoria Antonia Martínez Casado abandonó la cátedra, quedando San Román como profesor encargado. En 1944 ingresó en el Cuerpo de Médicos de Aguas Minero-Medicinales y, en una nueva oposición en 1947, ganó la cátedra de Hidrología Médica. El antiguo proyecto de creación de un instituto que cubriese las funciones de escuela de especialización y centro de investigación fue parcialmente satisfecho por el Consejo Superior de Investigación Científica al transformar contracorriente la sección de Hidrología existente en el Patronato "Santiago Ramón y Cajal" en el Instituto "Alfonso de Limón Montero" en 1952, cuya dirección pasó a 
ocupar. No obstante, los enfrentamientos con García Ayuso llevaron a una manifiesta separación entre cátedra/instituto y Cuerpo/Sociedad. Procedente San Román de la investigación en radiología y cáncer, consolidó también en el instituto y en la cátedra nuevas líneas de investigación en reumatología y mantuvo la hidrología experimental en una época de precariedad económica en la institución.

Oficio del Ilmo. Sr. Director General de Enseñanza Universitaria dirigido al Ilmo. Sr. Decano de la Facultad de Medicina, en el que se comunica que se ha resuelto que .... de dichas dotaciones se aplique una a dotar la Cátedra de "Hidrología Médica" de la Facultad de Medicina de la Universidad de Madrid. 14 de agosto de 1945

Archivo Facultad de Medicina. UCM

History of the International Society of Medical Hydrology and Climatolog (19221966) / M. Messini, with the collaboration of B. Messina and L. Dann Treves Pisa : Nistri-Lischi, imp. 1966

197 p. : il. ; $25 \mathrm{~cm}$

Sign: DE615.838(091)

Biblioteca Facultad de Medicina. UCM

Papeleta con la calificación de Sobresaliente en la asignatura de Histología, firmada por el catedrático Cajal.

Metabolismo y rayos $X$ : modificaciones que experimentan los componentes de la sangre y orina en los cancerosos sometidos a la acción de los rayos X/José de San Román y Rouyer.

Tesis inédita de la Universidad Central (Madrid), Facultad de Medicina, 1931

Sign. M1192

Servicio de Tesis doctorales. Biblioteca Universidad Complutense

"Importancia de la cura balnearia en los tiempos actuales" / José San Román y Rouyer

En: Clínica y Laboratorio : revista mensual española de ciencias médicas / Asociación Nacional de Prensa Médica

Zaragoza: [s.n.], 1905-1964

1942, XXXIII, p. 395-405

Sign.: PP 0MED

Biblioteca Facultad de Medicina. UCM 
"Ensayos de hidrología experimental" / José San Román y Rouyer

En: Medicina: revista mensual de ciencias médicas

1942, p.197

Madrid: [s.n.], 1928-1983

Sign.: PP 0MED

Biblioteca Facultad de Medicina. UCM

Hidrología médica / por José de San Román y Rouyer

Barcelona [etc.]: Salvat, 1945

403 p. : il. ; $23 \mathrm{~cm}$

Sign.: DE615.838SAN

Biblioteca Facultad de Medicina. UCM

Figura 13 - Portada

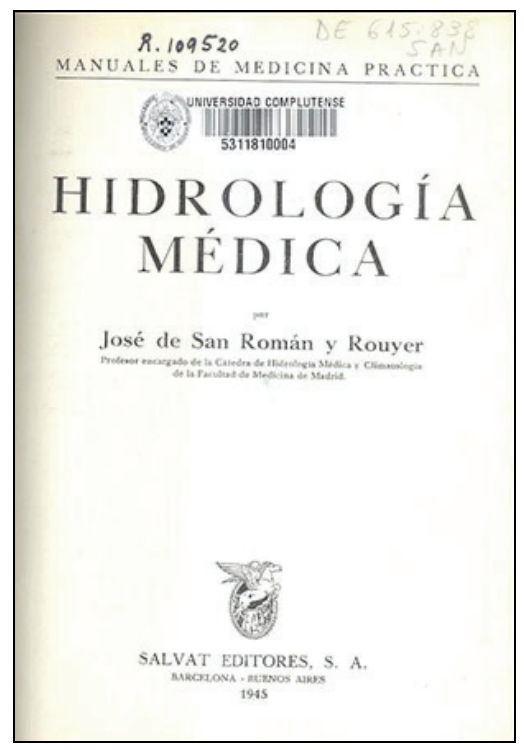

\section{Transiciones: Antonio Castillo de Lucas}

La vinculación personal del Instituto a la persona de José San Román llevó a su desaparición cuando éste falleció en 1961. Como profesor encargado de la cátedra quedó Antonio Castillo de Lucas, quien desempeñaba desde 1949 el puesto de Profesor Adjunto. Castillo no pertenecía al cuerpo de Médicos de Aguas MineroMedicinales y sólo en 1962 solicitó su pertenencia a la Sociedad Española de Hidrología Médica. La vulnerabilidad de la disciplina se ponía nuevamente de manifiesto, pero el decanato de Benigno Lorenzo Velázquez, catedrático de Farma- 
cología, posibilitó la rápida convocatoria de la oposición para cubrir la cátedra vacante. Aunque Castillo de Lucas había sido redactor jefe de los Anales Hispanoamericanos de Hidrología Médica y Climatología, revista del Instituto entre 1954 y 1958, y estaba publicando algunos trabajos pedagógicos sobre la Hidrología Médica, decidió no presentarse a las oposiciones a cátedra en 1963. Su labor siguió centrada en su importante investigación como paremiólogo.

"El Doctor Alfonso Limón Montero. Su vida y su obra" / Antonio Castillo de Lucas En: Anales Hispanoamericanos de Hidrología Médica y Climatología / Consejo Superior de Investigaciones Cientificas

Madrid: Consejo Superior de Investigaciones Científicas, 1954-1958

I, 1954, p. 11-54

Sign.: HE 1049

Biblioteca Facultad de Medicina. UCM

"Indicaciones hidroclimáticas" / por el Dr. Antonio Castillo de Lucas (Esquema de Clínica Hidrológica, en las enfermedades de los aparatos digestivo, respiratorio, circulatorio, urinario, sistema nervioso, endocrinopatías, afecciones de la piel y sífilis, ginecopatías y procesos quirúrgicos)

En: Guía Oficial de Balnearios y aguas minero-medicinales de España - 194647. p. 19-64

Cedida por el Profesor Castillo Ojugas

Figura 14 - Cubierta

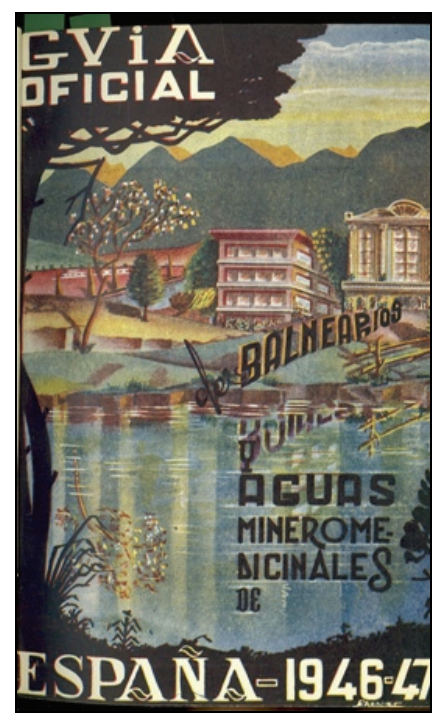


Cursillo de hidroclimatología médica / Antonio Castillo de Lucas; prólogo de Tomás Cerviá

Tenerife : Instituto de Fisiología y Patología Regional de Tenerife, 1962

155 p. : il. ; $24 \mathrm{~cm}$

Sign.: DE615.838CAS

Biblioteca Facultad de Medicina. UCM

"Indicaciones balnearias en el reumatismo" / por El Prof. A. Castillo de Lucas En: Cursillo de clínica hidrológica del reumatismo (artrosis): 26 de abril al 11 de mayo de 1962 / bajo la dirección de A. Castillo-Ojugas. Madrid: Universidad, 1962 p. 103-109

Sign.: 616.72-002.77-08 CUR

Biblioteca Facultad de Medicina. UCM

La medicina popular española / [Joaquín Fernández García, Antonio Castillo Ojugas editores]; prólogo de Pedro Laín Entralgo

Oviedo: Imp. D.G.M., D.L. 1998

Trabajos dedicados al Dr. D. Antonio Castillo de Lucas en el centenario de su nacimiento

Sign.: 61(05;4)MED

Biblioteca Facultad de Medicina. UCM

\section{Nuevas orientaciones: Manuel Armijo Valenzuela y la Escuela de Hidro- logía Médica}

Catedrático de Farmacología en la Universidad de Valladolid, médico del Cuerpo de Médicos Inspectores Baños, recién nombrado presidente de la Sociedad Española de Hidrología Médica y artífice de su nueva publicación (el Boletín de la SEHM), Manuel Armijo Valenzuela (1917- ), nuevo catedrático de Hidrología Médica tras la oposición de 1963, representaba en esos momentos la retomada relación entre Sociedad, clínica balnearia y cátedra, pero también entre farmacología e hidrología. Su labor, desarrollada también como Jefe de Sección de Farmacología del CSIC, propició un nuevo dinamismo investigador y abrió líneas experimentales en la cátedra. Durante sus años como catedrático fue decisiva la creación de la Escuela Profesional de Hidrología Médica e Hidroterapia, aprobada en 1968 (aunque puesta en funcionamiento diez años más tarde) con la que se realizaba la antigua aspiración de una formación especializada para los médicos de balnearios. Académico de número de la Real Academia Nacional de Medicina desde 1975, forma parte de las más relevantes sociedades hidrológicas internacionales. 
Estatutos de la escuela profesional de Hidrología médica e Hidroterapia de la facultad de Medicina de la Universidad de Madrid, con visto bueno del decano de la Facultad. Cátedra de Hidrología medica. Prof. Dr. M. Armijo Valenzuela.13 de marzo de 1968

Archivo de la Facultad de Medicina. UCM

Oficio trasladando al Rector de la Universidad de Madrid el Dictamen del Consejo Nacional de Educación por el que se aprueba la Creación de la Escuela Profesional de Hidrología médica y fisioterapia. Ministerio de Educación y Ciencia. Dirección de enseñanza superior de investigación. Subdirección de Enseñanza Universitaria.

5 de diciembre de 1968.

Archivo de la Facultad de Medicina. UCM

Programa de Hidrología Médica / por el Prof. Dr. M. de Armijo Valenzuela Universidad de Madrid. Facultad de Medicina, 1971

"El producto líquido de la venta de este programa se destina al mejoramiento de la enseñanza práctica de la Hidrología Médica"

Archivo Facultad de Medicina. UCM

Boletín Informativo. Instituto de España. Real Academia Nacional de Medicina. №246, año 1975

Recorte de prensa con fotografía del Prof. Armijo y una breve reseña biográfica

"Acción de las aguas minero-medicinales sobre el crecimiento y sobre la hepatotoxicidad del tetracloruro de carbono en las ratas (Aguas de Lanjarón, Cofrentes y Carballino)" / M. Armijo Valenzuela y D. Aguirre Miqueo

En: Archivos del Instituto de Farmacología Experimental / CSIC, Instituto de Farmacología Experimental, Sección de Madrid

1963, XI, 1, 95-106

Madrid: Consejo Superior de Investigaciones Científicas, 1949-1975

Sign.: PP 0MED

Biblioteca Facultad de Medicina. UCM

Compendio de hidrología médica / M. de Armijo Valenzuela

Barcelona [etc.]: Ed. Científico-Médica, 1968

XV, 484 p. : il. ; $25 \mathrm{~cm}$

Sign.: FR615.838ARM

Biblioteca Facultad de Medicina. UCM 
"Acción del agua radiactiva sobre el contenido de catecolaminas en tejido cerebral y aurícula de cobaya" / M. Armijo Valenzuela y A. Velasco Martín

En: Archivos del Instituto de Farmacología Experimental / CSIC, Instituto de

Farmacología Experimental, Sección de Madrid

1971, v. XXIII, fasc. 1 y 2, 107-8

Madrid: Consejo Superior de Investigaciones Científicas, 1949-1975

Sign.: PP 0MED

Biblioteca Facultad de Medicina. UCM

"Acción del agua radiactiva sobre el crecimiento de cultivos de corazón embrionario" / M. Armijo [et al.]

En: Archivos de la Facultad de Medicina de Madrid / Universidad Complutense

1972, v. XXII, n. 4, 185-9

Madrid: Universidad Complutense, 1961-1981

Sign.: PP 0MED

Biblioteca Facultad de Medicina. UCM

Sauna / Manuel Armijo Valenzuela, Josefina San Martín Bacaicoa

Madrid: Oteo, 1976

97 p. : il. ; $21 \mathrm{~cm}$

Sign.: DE615.838ARM

Biblioteca Facultad de Medicina. UCM

Figura 15 - Cubierta

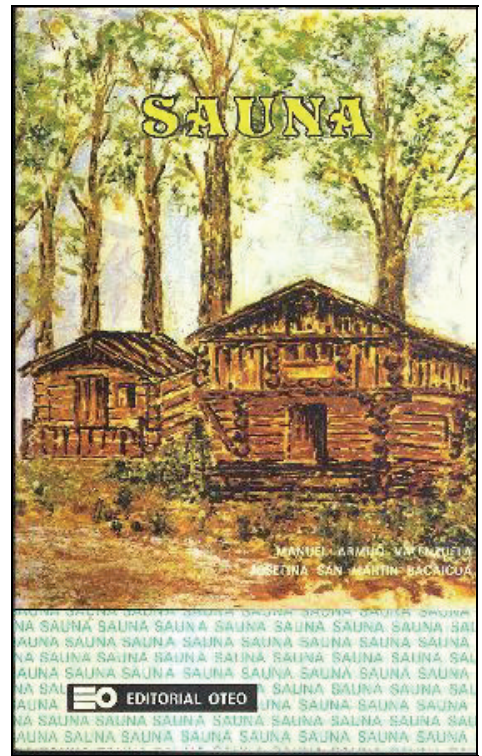


"La cura balnearia en el envejecimiento condro-articular" / M. Armijo Valenzuela

En: Anales de la Real Academia Nacional de Medicina (Madrid)

1981, t. XCVIII, fasc. 4, 603-26

Madrid: Real Academia Nacional de Medicina, 1879-

Sign.: PP 0MED

Biblioteca Facultad de Medicina. UCM

La Salud por las aguas termales / M. Armijo Valenzuela y J. San Martín Baicoa Madrid: EDAF, D.L.1984

120 p. ; $21 \mathrm{~cm}$

Sign. :DE615.838ARM

Biblioteca Facultad de Medicina. UCM

Hidrología / Director: Alberto Berguer, autores M. Armijo Valenzuela [et al.]

[Madrid]: Consejo General de Colegios Médicos de España, D.L. 1990

Serie monográfica de especialidades médicas; 45

118 p. ; $27 \mathrm{~cm}$

Sign.: FR615.838:378HID

Biblioteca Facultad de Medicina. UCM

Curas balnearias y climáticas: talasoterapia y helioterapia / Manuel Armijo Valenzuela, Josefina San Martín Bacaicoa

Madrid : Editorial Complutense, 1994

[1a ed.]

IX, 688 p. : il. ; $25 \mathrm{~cm}$

Sign.: FR615.839ARM

Biblioteca Facultad de Medicina. UCM

"Estudios sobre el balneario de Arnedillo" / J. M. López de Azcona ... [et al.]

En: Monografias de la Real Academia Nacional de Farmacia, memoria ${ }^{\circ}$ 14, 1988 Madrid: Real Academia de Farmacia, 1988

68 p. : il. ; $24 \mathrm{~cm}$

Biblioteca Facultad de Medicina. UCM

\section{Josefina San Martín Bacaicoa: internacionalización}

Médica con triple especialización en Rehabilitación, Reumatología e Hidrología Médica, Josefina San Martín ganó la oposición a la cátedra en 1986. Toda su labor docente, investigadora y asistencial ha estado relacionada con las aguas mineromedicinales desde 1971. Ha formado a investigadores (con más de veinte tesis y tesinas) y especialistas, asumiendo en 1994 la dirección de la Escuela Profesional de Hidrología Médica e Hidroterapia. La conjunción de sus especializaciones la han 
hecho experta en hidroterapia e hidrocinesiterapia. Sus líneas de investigación han sido diversas y han incluido estudios analíticos y de experimentación animal, trayectoria que se ha reflejado en abundantes publicaciones e intervenciones en congresos. Desde el inicio de su carrera profesional ha participado en reuniones científicas internacionales, frecuentemente como ponente invitada, y ha sido miembro de sociedades como la International Society of Medical Hydrology and Climatology (que la nombró miembro de honor en 1998) o la International Society of Hydrothermal Technique que le concedió en 1996 su medalla de oro «Prix du Thermalisme». Es Académica Correspondiente de las Reales Academias Nacionales de Medicina y de Farmacia.

Relación entre la conductividad especifica y la densidad relativa de las aguas minero-medicinales y su residuo seco / Josefina San Martín Barcaicoa ; [director] Manuel de Armijo Valenzuela

Universidad Complutense de Madrid. Facultad de Medicina. Tesis inéditas

$134 \mathrm{f.} ; 28 \mathrm{~cm}$

Sign.: T 7189

S. Tesis Doct.orales Inéditas

"Contenido en ion fluoruro de algunas aguas mineromedicinales utilizadas frecuentemente como "aguas de mesa" / M. Armijo, Josefina Martín y F. Armijo

En: Anales de Bromatología, 1976, XXVIII, 2, 179-92

Madrid : [s.n.], 1976

Sign.: Fo 24(9)

Biblioteca Facultad de Medicina. UCM

"Estudio de las fuentes minerales de la provincia de Teruel" / J. San Martín, J. y F. Armijo

En: Teruel / CSIC, Instituto de Estudios Turolenses

1986, p. 83-117

Teruel: Consejo Superior de Investigaciones Científicas, 1949-

Sign.: PP 0FARBOT

Facultad de Farmacia. Dpto. Botánica. UCM

"Acción diurética y ponderal de las aguas débilmente mineralizadas" / J. San Martín En: Anales de la Real Academia Nacional de Medicina (Madrid)

1989, t. CVI, fasc. 4, 567-84

Madrid: Real Academia Nacional de Medicina, 1879-

Sign.: PP 0MED

Discurso de ingreso en la Real Academia Nacional de Medicina

Biblioteca Facultad de Medicina. UCM 
Fotografía con la toma de posesión como Académica de la Real Academia Nacional de Farmacia. Entrega el nombramiento el Excmo. Sr. Don Julio Rodríguez Villanueva, Presidente de la Real Academia Nacional de Farmacia.

"Evaluación de la utilidad de la cura balnearia" / J. San Martín Bacaicoa.

En: Boletín de la Sociedad Española de Hidrología Médica

1991, VI, n.1, 9-11

Madrid: Sociedad Española de Hidrología Médica, 1962-

Sign.: PP 0MED

Biblioteca Facultad de Medicina. UCM

Fotografía con la toma de posesión como Académica de la Real Academia Nacional de Medicina. Entrega el nombramiento el Excmo. Sr. Don José Botella Llusiá

"Sulfur water flora a S.E.M. and X-Ray microanalitical study" / J. San Martín Bacaicoa, F. Maraver Eyzaguirre, V. Crespo Ferrer

En: 7 Giornate Mondiali del Termalismo e Conferenza Permanente dell'O.M.Th., Verona 22-25 Maggio 1987 / Organizzate dall'O.M.Th. dall'Associazione Italiana di Tecnica Idrotermale e dalla Sezione Triveneta dell'A.I.T.I. : atti del convengo Verona: [s.n.], imp. 1990

"Health Resort Medicine Integration in Universities" / J. San Martín Bacaicoa En: 32nd World Congress of the I.S.M.H. April 1994. Bad Wörishofen (Germany). Sign.: D618.838 INT

Biblioteca Facultad de Medicina. UCM

"Talasoterapia : proyección terapéutica actual" / Josefina San Martín Bacaicoa En: Anales de la Real Academia Nacional de Medicina, tomo CXII, cuaderno segundo, 1995

Madrid : Real Academia Nacional de Medicina, 1879-

Sign.: PPOMED

Biblioteca Facultad de Medicina. UCM

"Aguas mineromedicinales y minerales naturales como agentes medicamentosos": discurso de ingreso como Académica Correspondiente en la Real Academia de Farmacia

En: Anales de la Real Academia de Farmacia, Vol. LXIII, Núm. 4, 1997

Madrid: Real Academia de Farmacia, 1940-2002

Sign.: PPOFAR

Biblioteca Facultad de Farmacia. UCM 
Figura 16 - Cubierta

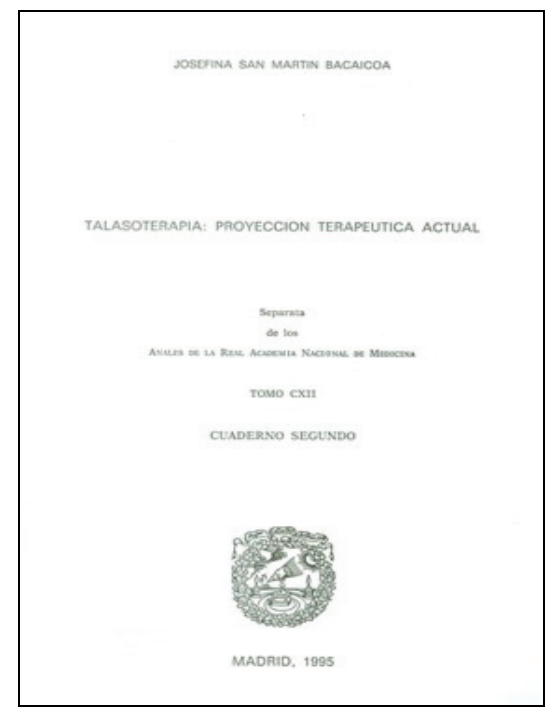

"Physiology of aquatic therapy: resistance factors" / San Martín Bacaicoa J En: 34th World Congres of the International Society of Medical Hydrology and Climatology: abstract book: Budapest, Héviz, Hungary, october 14-19, 2002 / organised by the ISMH and the Hungarian Balneological Association with cooperation of the Hungarian Society for Rehabilitation of the Disabled [Budapest]: the ISMH, 2002

209 p. ; $21 \mathrm{~cm}$

Sign.: DE615.838(063)

Biblioteca Facultad de Medicina. UCM

Anales de la Real Academia Nacional de Farmacia. Vol. LXXXV, $\mathrm{n}^{\circ}$ extraordinario, 2009

"Estudio de la acción terapéutica de las aguas del Balneario de Alicún de las Torres" / Josefina San Martín Bacaicoa, Agustín Valero Castejón

En: Anales de la Real Academia Nacional de Farmacia, Vol. LXXV, Núm. Extraordinario, 2009

Madrid: Real Academia Nacional de Farmacia, 2002-

Sign.: PPOFAR

Biblioteca Facultad de Farmacia. UCM 
"Acción terapéutica de las aguas del Balneario de Puente Viesgo" / San Martín Bacaicoa, J. y Valero Castejón, A.

En: Anales de la Real Academia Nacional de Farmacia, Vol. LXXIII, Núm. Extraordinario, 2007

Madrid: Real Academia Nacional de Farmacia, 2002-

Sign.: PPOFAR

Biblioteca Facultad de Farmacia. UCM

\section{La docencia de la Hidrología Médica: de la estructura departamental a los retos del espacio europeo}

\subsection{Francisco Maraver Eyzaguirre: relaciones institucionalizadas}

Profesor Titular de Hidrología Médica desde 1987, es Director de la Escuela Profesional de Hidrología Médica e Hidroterapia desde el año 2000. Desde 1981, a través de su actividad asistencial y docente ha formado a investigadores (con la dirección de más de setenta trabajos académicos) y médicos especialistas. Ha asumido la adaptación de la disciplina al espacio europeo de educación superior, incorporándola también a la formación de grado en distintas titulaciones. En su labor investigadora han destacado sus estudios sobre los productos derivados de las aguas mineromedicinales, gases y peloides, plasmados en publicaciones de algunas de las cuales ha sido fundador y director, como los Anales de Hidrología Médica y la serie de monografías Balnea. Ha promovido relaciones institucionalizadas con relevantes organismos a nivel nacional e internacional, en Europa e Iberoamérica, que han permitido la proyección de la cátedra. Esta función se relaciona con una intensa labor asociativa nacional -es Presidente de la Sociedad Española de Peloides Termales y del Comité Científico del Instituto de Investigación Agua y Salud- e internacional, con la membresía de honor de diversas asociaciones y el reconocimiento por la Sociedad Francesa de Hidrología y Climatología Médica con su Medalla de Honor. Es el Presidente del 38th World Congress ISMH que se celebrará en Lanjarón-Granada en junio de 2012.

Vademécum de aguas mineromedicinales españolas / Francisco Maraver (Director Coordinador)

[Madrid] : Instituto de Salud Carlos III, [2003]

1 v. (pág. var.) ; $24 \mathrm{~cm}$

Portada y texto contrapuestos en inglés

Sign.: FR 615.848(460)VAD

Biblioteca Facultad de Medicina. UCM 
Figura 17 - Cubierta

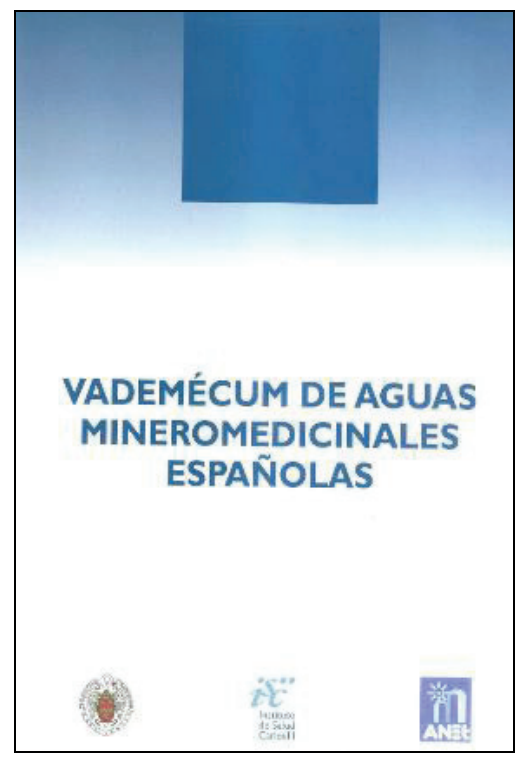

Anales de hidrologia médica

Madrid: Publicaciones Universidad Complutense de Madrid, 2006-

ISSN 1887-0813

Sign.: PP0MED

Biblioteca Facultad de Medicina. UCM

Balnea / Francisco Maraver Ayzaguirre, dir.

Madrid: Publicaciones Universidad Complutense de Madrid, 2006-

Balnea es una serie de monografías relacionadas con la revista Anales de Hidrología Médica. El primer número aparece como Anejo I y el siguiente cambia la designación por número 2 .

Los números de esta publicación son monográficos que se pueden localizar de forma individual, buscando su título o autor propios, en los índices correspondientes Sign.: 615.838 CAT

Biblioteca Facultad de Medicina. UCM

"Las aguas minerales naturales" / Francisco Maraver Eyzaguirre

En: El agua mineral natural en la infancia / Asociación Española de Pediatría, Instituto de Investigación Agua y Salud.

Madrid: CTO, D.L. 2009, p. 12-34

Sign.: Fo 2009(7)

Biblioteca Facultad de Medicina. UCM 
Vademécum II de aguas mineromedicinales españolas / Francisco Maraver Eyzaguirre, Francisco Armijo Castro

Madrid: Editorial Complutense, 2010

365 p. : il. ; $24 \mathrm{~cm}$.

Sign.: FR615.838(460)MAR

Biblioteca Facultad de Medicina. UCM

"Estudio de las aguas minerales de la provincia del Neuquén, Argentina"

En: $1^{\circ}$ Congreso Internacional de Termalismo, Neuquén, octubre 2011. [Neuquén]: [s. n.], 2011. P. 31

Sign.: DE615.838CON

Biblioteca Facultad de Medicina. UCM

\subsection{Francisco Armijo Castro: nuevas técnicas analíticas}

Químico, diplomado en Plásticos y Caucho, especialista en Química Médica y doctor en Farmacia. Desde 1969 vinculado a la Cátedra de Hidrología Médica como profesor ayudante de clases prácticas, asociado y titular y, desde 2001, es subdirector de la Escuela Profesional de Hidrología e Hidroterapia. Su actividad se desarrolla en el ámbito del análisis físico químico del agua y de los peloides. Siempre ha compatibilizado la labor universitaria con puestos como técnico de I+D en la industria farmacéutica y como director técnico en empresas españolas y multinacionales dedicadas a la instrumentación de laboratorio. Esta doble actividad le llevó a poner a punto el laboratorio de la Cátedra con técnicas pioneras como los electrodos específicos, la absorción atómica y la cromatografía iónica que ha permitido realizar los análisis de los balnearios españoles, de las aguas envasadas, de las potables de ciudades españolas, así como de manantiales para su utilización como aguas mineromedicinales. Desde 1990 ha participado en decenas de proyectos de investigación financiados por diferentes entidades, en colaboración con otras facultades y universidades, habiendo sido ponente en congresos nacionales e internacionales del ámbito de la Hidrología y la Farmacia Galénica.

Viajes de agua: las aguas en el pirineo aragonés a través de sus viajeros / Francisco Armijo de Castro

Jaca : Pirineum editorial , D. L. 2007

197 p. : il. ; $24 \mathrm{~cm}$

Sign.: FR615.838ARM

Biblioteca Facultad de Medicina. UCM 
Figura 18 - Cubierta

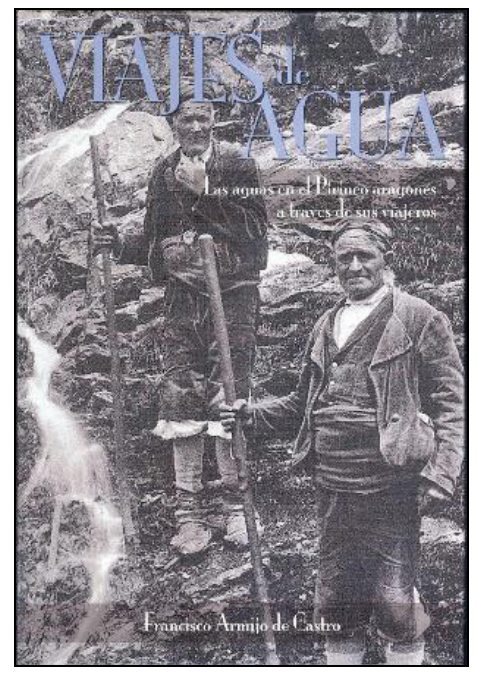

"Los peloides terapéuticos: Experiencia de la cátedra de hidrología Médica" En: Termatalia / Sesión científica sobre Termalismo de la Real Academia de Medicina y Cirugía de Galicia, Madrid 20 de enero de 2009. P. 191-222 Ourense : Xunta de Galicia, Dirección Xeral de Saúde Pública, D. L. 2009 Sign.: DE615.838TER

Biblioteca Facultad de Medicina. UCM

"Cien años de análisis de las aguas medicinales". Francisco Armijo Castro En: Balnea. 2012. Número 5. Serie de Monografías. Publicaciones Universidad Complutense de Madrid Biblioteca Facultad de Medicina. UCM

\section{La contribución a la Hidrología Médica desde otras ciencias}

Si el balneario es un espacio de encuentro de diferentes colectivos con variados intereses, el agua mineromedicinal también ha supuesto siempre un reto para diferentes disciplinas científicas. Química y Farmacia pretendían comprender la acción de las aguas a través del análisis y así dar el decisivo paso para reproducirlas artificialmente. Biología y Climatología truncaban en buena medida este sueño de la cura hidromineromedicinal fuera del balneario al mostrar la importancia de los microrganismos y de la Geografía Médica. Geografía y Cartografía representaron en el mapa la distribución de las fuentes e interpretaron su localización por zonas. Por su parte, la Geología y la Ingeniería de Minas se convirtieron en firmes aliadas 
para el descubrimiento, mejor captación y protección de los manantiales. Todas condujeron a una visión compleja de la terapéutica con aguas minerales que la Hidrología Médica integró como disciplina.

\subsection{El misterio de las aguas mineromedicinales: la Química}

Teoremas i problemas para examinar, i saber usar qualesquiera aguas minerales ... i por medio de ellos se averigua ... la naturaleza, i virtudes del agua mineral del Pilar de la ciudad de Chinchilla, i de la acreditada de Puerto Llano / dispuestos por D. Antonio Capdevila

En Madrid: por Andrés Ortega, 1775

[8], $45 \mathrm{p} . ; 4^{\circ}$

Sign.: BH MED 16443(2) ; BH MED Foll.318 ; BH MED 11487(2)

B.Histórica-F.Antiguo (M). UCM

Figura 19 - Cubierta

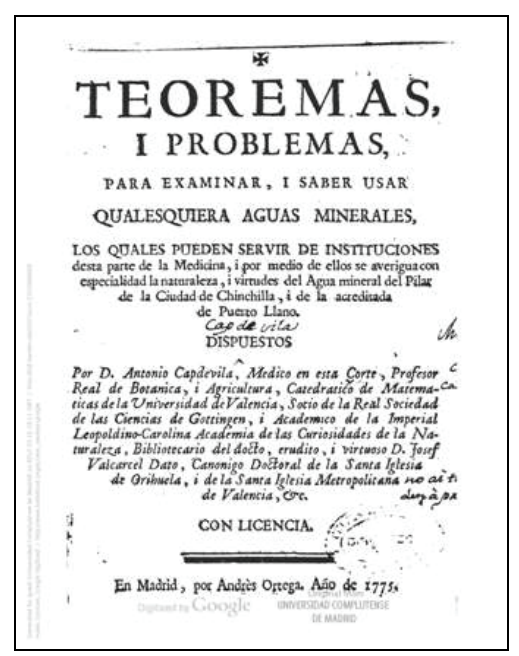

Instruccion sobre el mejor metodo de analizar las aguas minerales, y en lo posible imitarlas / por ... Pedro Gutierrez Bueno ...

En Madrid: en la Imprenta Real, MDCCLXXYII [i.e. 1782]

[4], 56 p. ; $8^{\circ}$

Sign.: BH MED 11987(1)

B.Histórica-F.Antiguo (M). UCM 
Tratado práctico de análisis quimica de las aguas minerales y potables: con las indicaciones de las fuentes de aguas mas notables de España, su composicion, enfermedades á cuya curacion se aplican, y número de enfermos que ña ellas acuden anualmente / por D. Antonio Casares ...

Madrid: Libreria de D. Angel Calleja, 1866

VIII, 271, [1] p. ; $21 \mathrm{~cm}$

Sign.: A54"18"C25a

Biblioteca de Farmacia. UCM

\subsection{La comprensión del terreno: la Geología y las aguas minero- medicinales}

Boletín de la Comisión del Mapa Geológico de España

Madrid: Imprenta y Fundicion de Manuel Tello ..., 1874-1910

Periodicidad anual

Sign.: PP 1606GEO

Biblioteca Facultad de Geológicas. UCM

Monografía de las aguas minerales y termales de España / Federico de Botella y de Hornos, Inspector General

Madrid: Imp. del Colegio Nacional de Sordomudos y de Ciegos, 1892

X, 143 p., [1] lám pleg. ; $34 \mathrm{~cm}$

Sign.: DA6732

Biblioteca Facultad de Medicina. UCM

Figura 20 - Cubierta

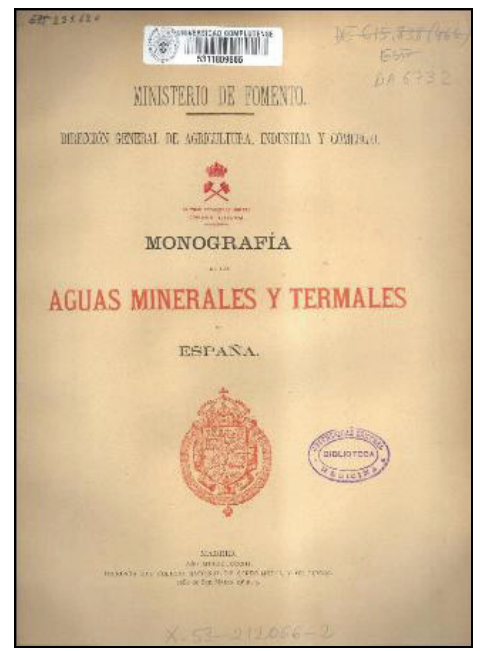




\subsection{El agua minero-medicinal como fármaco}

Estudios sobre el Balneario de Archena / Comisión para el Estudio de las Aguas Medicinales

Madrid: Real Academia de Farmacia, 1986

52 p. : il. ; $24 \mathrm{~cm}$.

Memoria ; 12

Sign.: D615.838AMM-12

Biblioteca Facultad de Farmacia

La contaminación del ambiente y su influencia en la vida: discurso leído en la solemne sesión inaugural del curso 1970-71[...] / por Juan Manuel López de Azcona

Madrid: Real Academia de Farmacia, 1971

59 p. ; $24 \mathrm{~cm}$.

Real Academia de Farmacia (España). Discursos de inauguración de curso Sign.: HIS F167

Biblioteca Facultad de Farmacia. UCM

\subsection{Geografía}

Atlas del diccionario geográfico-estadístico-histórico de España y sus posesiones de ultramar. Islas Canarias [Material cartográfico] / formado el mapa en vista de diferentes documentos por el Teniente Coronel Capitán de Ingenieros D. Francisco Coello y Pascual Madoz

1 mapa en 2 h. : col., montado sobre tela ; 99x74 cm., pleg. $21 \times 14 \mathrm{~cm}$

Madrid, 1849

Sign.: DA 2535

Biblioteca Facultad de Medicina. UCM

Cartografia hispano-cientifica ó sea los mapas españoles en que se representa á España bajo todas sus diferentes fases / su autor... Francisco Jorge Torres Villegas Madrid: Imprenta de D. Ramon Ballone, 1857

2 t. : map.; $30 \mathrm{~cm}$

Sign.: A9"18" T68f Tomo I

Biblioteca Facultad de Farmacia. UCM 
Figura 20 - Portada

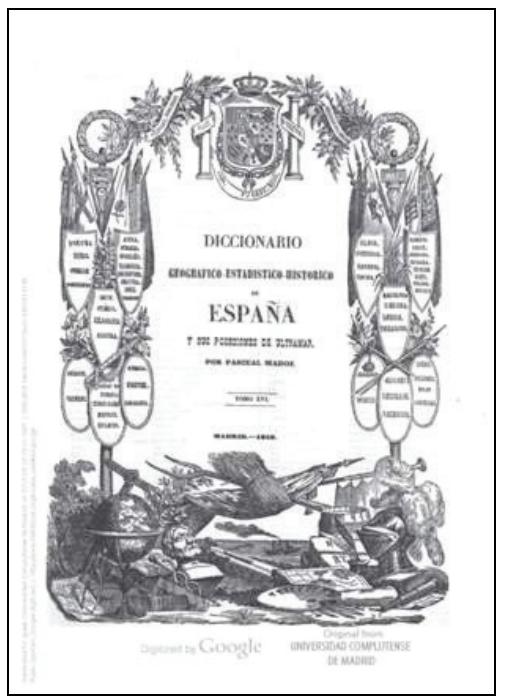

Fuera de las secciones en vitrina aparte:

Baños termo-minerales de Fortuna: [memoria descriptiva] / Juan Cascales Font [4] h., [3] h. de tabl. ; $30 \mathrm{~cm}+$ plano

Acompaña plano: "Provincia de Murcia, Baños Termales de Fortuna, plano topográfico de la situación de los nuevos baños y terrenos adyacentes", firmado en Murcia 26 de Agosto de 1868. En tinta negra y roja. Escala 1:100. 36 x $63 \mathrm{~cm}$ Sign.: Ca2794(14)

Biblioteca Facultad de Medicina. UCM

Memoria descriptiva y planos del establecimiento de Baños del Real Sitio del Solán de Cabras / Alejandro Sureda

[4] p. ; $27 \mathrm{~cm}+$ plano

Acompaña plano: "Balneario de Solán de Cabras", firmado en Madrid, el 16 de mayo de 1868, por Alejandro Sureda. En tinta negra, azul y rojo

Sign.: Ca 2727(4)

Biblioteca Facultad de Medicina. UCM 
Figura 21 - Plano

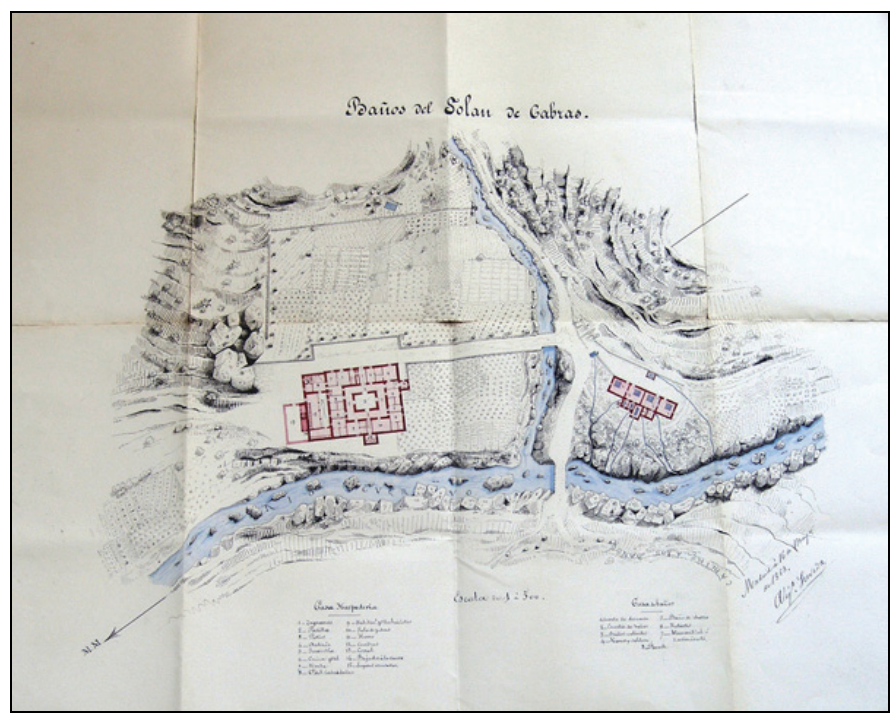

Balneario de San Telmo en Jerez de la Frontera: aguas cloruradas sódicas sulfurosas, temporada 10 de junio a 30 de septiembre / el médico director Joaquín Aleixandre

Madrid: Imp. de Ricardo Rojas, 1900

31 p. : il. ; $17 \mathrm{~cm}+[2]$ h. manuscritas

Incluye plano y fotografías del interior y exterior del establecimiento

Sign.: Ca2847(15)

Biblioteca Facultad de Medicina. UCM

Reproducción de la Pátera de Otañes, Descubierta en el siglo XVII en Otañes (Cantabria). Bien de Interés cultural en el año 2000- Parece ser una ofrenda a la ninfa de la fuente termal del Santuario de Umérita. Representa la ninfa Salus Umeritana haciendo brotar agua salutífera de una roca.

Medalla de Honor y otras medallas conmemorativas de distintos eventos de Hidrología Médica.

16. La exposición virtual http://www.ucm.es/BUCM/med/51068.php

El motivo de realizar la versión virtual de esta exposición fue aprovechar las posibilidades que ofrece este formato. A la hora de preparar la exposición física nos 
encontramos con varias limitaciones que podían quedar subsanadas o mejoradas con la exposición virtual, como eran:

a) La limitación de espacio para exhibir los textos y documentos en las vitrinas, lo que nos obligaba a hacer una selección de los mismos. Sin embargo, la exposición virtual nos permitía mostrar un mayor número de todos ellos.

b) Las limitaciones impuestas por la seguridad y conservación de algunos textos (incunables y obras del siglo XVI y XVII) condicionaban su exhibición, cuestión que en la versión virtual no ofrece impedimentos.

c) La exposición tiene una fecha de clausura y finalización. Sin embargo, la exposición virtual en soporte online puede tener un carácter permanente en el tiempo lo que la convierte en un portal de información duradero sobre la investigación y los estudios llevados a cabo en los últimos cien años por la Cátedra de Hidrología Médica de la Facultad de Medicina de la Universidad Complutense de Madrid. 